\begin{tabular}{|c|l|}
\hline Title & $\begin{array}{l}\text { Changes in the spontaneous calcium oscillations for the development of the preconditioning-induced ischemic tolerance } \\
\text { in neuron/astrocyte co-culture. }\end{array}$ \\
\hline Author(s) & Tanaka, Motoki; Kawahara, Koichi; Kosugi, Tatsuro; Y amada, Takeshi; Mioka, Tetsuo \\
\hline Citation & $\begin{array}{l}\text { Neurochemical Research, 32(6), 988-1001 } \\
\text { https://doi.org/10.1007/311064-006-9259-8 }\end{array}$ \\
\hline Issue Date & 2007-06 \\
\hline Doc URL & http://hdl.handle.net/2115/25393 \\
\hline Rights & The original publication is available at www.springerlink.com \\
\hline Type & article (author version) \\
\hline File Information & NR32-6.pdf \\
\hline
\end{tabular}

Instructions for use 


\section{Changes in the spontaneous calcium oscillations for the development of the preconditioning-induced ischemic tolerance in neuron/astrocyte co-culture}

Motoki Tanaka, Koichi Kawahara, Tatsuro Kosugi, Takeshi Yamada and Tetsuo Mioka

Laboratory of Cellular Cybernetics, Graduate School of Information Science and

Technology, Hokkaido University, Sapporo 060-0814 Japan

Running title: calcium oscillations and ischemic tolerance

Corresponding Author:

Koichi Kawahara, Ph. D.

Professor of the Laboratory of Cellular Cybernetics

Graduate School of Information Science and Technology

Hokkaido University

Sapporo 060-0814

Japan

TEL \& FAX: +81-11-706-7592

E-mail: kawahara@cellc.ist.hokudai.ac.jp 


\section{Abstract}

Spontaneous $\mathrm{Ca}^{2+}$ oscillations are believed to contribute to the regulation of gene expression. Here we investigated whether and how the dynamics of $\mathrm{Ca}^{2+}$ oscillations changed after sublethal preconditioning (PC) for PC-induced ischemic tolerance in neuron/astrocyte co-cultures. The frequency of spontaneous $\mathrm{Ca}^{2+}$ oscillations significantly decreased between 4 and $8 \mathrm{~h}$ after the end of PC in both neurons and astrocytes. Treatment with 2-APB, an inhibitor of IP3 receptors, decreased the oscillatory frequency, induced ischemic tolerance and a down-regulation of glutamate transporter GLT-1 contributing to the increase in the extracellular glutamate during ischemia. The expression of GLT-1 is known to be up-regulated by PACAP. Treatment with PACAP38 increased the oscillatory frequency, and antagonized both the PC-induced down-regulation of GLT-1 and ischemic tolerance. These results suggested that the PC suppressed the spontaneous $\mathrm{Ca}^{2+}$ oscillations regulating the gene expressions of various proteins, especially of astrocytic GLT-1, for the development of the PC-induced ischemic tolerance. 
Key words: spontaneous $\mathrm{Ca}^{2+}$ oscillations, ischemic tolerance, GLT-1, PACAP, oxygen and glucose deprivation, neuron/astrocyte co-cultures.

\section{Abbreviations}

PC

Preconditioning

$\mathrm{CM}$

Conditioned medium from astrocytes cultured for more than 2 weeks

GFAP Glial fibrillary acidic protein

OGD Oxygen-glucose deprivation

NADH Nicotinamide adenine dinucleotide

2-APB 2-Aminoethyl diphenylborinate

IP3 Inositol 1, 4, 5-trisphosphate

PACAP Pituitary Adenylate Cyclase Activating Polypeptide 


\section{INTRODUCTION}

Spontaneous oscillations of the intracellular concentration of calcium $\left(\mathrm{Ca}^{2+}\right.$ oscillations) are a signaling system in cells. The oscillations play functional roles in neuronal migration and development, axonal and dendritic growth, and the formation of the neuronal network [1-5]. In addition, recent studies have reported that frequency is more important than amplitude, and the frequency of $\mathrm{Ca}^{2+}$ oscillations regulates the efficiency of the gene expression of specific proteins [6-8]. Previous studies have revealed that the oscillatory frequency is regulated by neurotransmitters, growth factors, and cytokines [9-11], suggesting that the intracellular $\mathrm{Ca}^{2+}$ oscillations are influenced by the external environment, and have the role of adapting cells to the external environment.

Ischemic tolerance is the phenomenon whereby a brief sub-lethal ischemia (preconditioning, PC) provides protection against the neuronal degeneration caused by a subsequent prolonged lethal ischemia [12-15]. Previous studies have demonstrated that the ischemic tolerance is induced about $24 \mathrm{~h}$ after the PC in vitro $[14,15]$ or after about $3 \mathrm{~d}$ in vivo [13], and 
the PC is believed to induce ischemic tolerance via the regulation of various genes and proteins important for neuronal protection [16-19]. The regulation of intracellular free $\mathrm{Ca}^{2+}$ has been intensively studied as a key factor in the induction of ischemic neuronal cell death or in the development of PC-induced ischemic tolerance. In addition, a number of studies have been performed on the dynamics of intracellular $\mathrm{Ca}^{2+}$ oscillations during either lethal ischemia or PC, but have reported contradicting results; some studies reported that the changes contribute to neurodegeneration [20-21], others that they contribute to neuroprotection [22-24]. And, the relationship between the changes in the dynamics of intracellular $\mathrm{Ca}^{2+}$ oscillations during the period between PC and lethal ischemia and the development of ischemic tolerance has not been studied in detail. There is a possibility that the changes in $\mathrm{Ca}^{2+}$ oscillation-regulated gene expression during the period between PC and lethal ischemia contributes to the development of ischemic tolerance.

Here we provide experimental evidence that PC-induced changes in spontaneous $\mathrm{Ca}^{2+}$ oscillations in neurons and/or astrocytes are crucially involved in the development of PC-induced neuronal ischemic tolerance 
by transiently down-regulating the expression of the astrocytic glutamate transporter GLT-1 in neuron/astrocyte co-cultures. 


\section{MATERIALS AND METHODS}

\section{Cell Culture}

Culture methods were described previously in detail [25-27]. In brief, neurons/astrocytes were prepared from 17-day-old embryonic rat cortices, digested with $0.02 \%$ papain (Boehringer, Germany) and mechanically dissociated, and grown in Dulbecco's modified Eagle's medium (DMEM; Gibco, Grand Island, NY), which was supplemented with 10\% heat-inactivated fetal bovine serum (FBS), 10\% Ham's F12, and $0.24 \%$ penicillin/streptomycin (culture medium). Cells were plated at a uniform density of $3.0 \times 10^{4}$ cells/cm ${ }^{2}$ onto poly-L-lysine $(100 \mu \mathrm{g} / \mathrm{ml})$ and Laminin $(20 \mu \mathrm{g} / \mathrm{ml})$-coated plastic dishes and maintained in a $5 \% \mathrm{CO}_{2}$ incubator at $37^{\circ} \mathrm{C}$. The cultures were fed a filtered $(0.22 \mu \mathrm{m}$; Millipore, Bedford, TX) conditioned culture medium (CM) twice a week. After 13-15 days, the neurons in these cultures sit on the top of a confluent monolayer of astrocytes. The experiments were performed using these cultures.

To obtain the CM [28], cells from the 17-day old embryonic rat cortices were plated onto $2 \%$ polyethyleneimine-coated 6-well dishes. 
The cultures were then fed a cooled culture medium and incubated for an additional day. The cultures became astrocyte-enriched with few neurons after more than 2 weeks. The CM for neuron/astrocyte co-cultures was conditioned by such cultures.

\section{Intracellular $\mathrm{Ca}^{2+}$ measurements and identification of astrocytes}

Intracellular $\mathrm{Ca}^{2+}$ was measured in both neurons and astrocytes after loading the cells with the $\mathrm{Ca}^{2+}$-sensitive fluorescent dye Fluo4-AM. Cell loading was performed using $5 \mu \mathrm{M}$ of Fluo4-AM in conditioned medium supplemented with $0.03 \%$ Pluronic F-127 for $50-60$ min at $37{ }^{\circ} \mathrm{C}$. Intracellular $\mathrm{Ca}^{2+}$ measurements were performed in a balanced salt solution (142 mM NaCl, $0.8 \mathrm{mM} \mathrm{MgSO}_{4}, 5.4 \mathrm{mM} \mathrm{KCl,} 1.0 \mathrm{mM} \mathrm{NaH} \mathrm{PO}_{4}$, $4 \mathrm{mM} \mathrm{NaHCO} 3,1.8 \mathrm{mM} \mathrm{CaCl}_{2}, 10 \mathrm{mM}$ HEPES, $0.01 \mathrm{mM}$ glycine, and 20 mM glucose). The fluorescence was excited at $480 \mathrm{~nm}$ and detected at $>$ $510 \mathrm{~nm}$ with a fluorescence microscope (Olympus, IX70). Images were acquired with integration times of $400 \mathrm{~ms}$ at intervals of $4 \mathrm{~s}$ and analyzed off-line (HAMAMATSU, Aquacosmos). The change in intracellular $\mathrm{Ca}^{2+}$ was estimated from the ratio of the fluorescence $\left(\mathrm{F} / \mathrm{F}_{0}\right) . \quad \mathrm{Ca}^{2+}$ spikes were 
defined as rapid elevations of $\Delta \mathrm{F} / \mathrm{F} 0 \geq 0.1$ or 0.2 . Although this threshold produced false positives in some cells in which noise base-line values were scored as transients, as determined by visual inspection, but in most cases this criterion represented $\mathrm{Ca}^{2+}$ spike correctly.

To distinguish them from neurons in the analysis of calcium oscillations, astrocytes were identified by immunostaining with antibodies against glial fibrillary acidic protein (GFAP, Sigma, 1:5000) after intracellular $\mathrm{Ca}^{2+}$ measurements. For labeling, a 1:200 dilution of Alexa Fluor 532 goat anti-mouse IgG (Molecular Probes) was used. The fluorescence was excited at $530 \mathrm{~nm}$ and detected at > $580 \mathrm{~nm}$ with a fluorescence microscope. We distinguished neurons and astrocytes by the images of Fluo- 4 and GFAP in the same regions in the co-cultures (Fig. 1A2 and A3).

\section{Oxygen-Glucose deprivation}

Cortical cultures were subjected to oxygen-glucose deprivation (OGD) injury using a protocol described previously [26, 29, 30]. In brief, cultures were placed in an anaerobic chamber containing the 
deoxygenation reagent and glucose-free balanced salt solution (BSS: 116 $\mathrm{mM} \mathrm{NaCl}, 0.8 \mathrm{mM} \mathrm{MgSO}$, $5.4 \mathrm{mM} \mathrm{KCl}, 1.0 \mathrm{mM} \mathrm{NaH} \mathrm{PO}_{4}, 26.2 \mathrm{mM}$ $\mathrm{NaHCO}_{3}, 1.8 \mathrm{mM} \mathrm{CaCl}_{2}$, and $0.01 \mathrm{mM}$ glycine) was added. Near anoxic conditions were achieved using an Anaero-Pack System (Mitsubishi Gas Chemical, Tokyo, Japan). To terminate OGD, cultures were carefully washed with glucose $(20 \mathrm{mM})$ containing BSS, and then incubated again in culture medium at $37{ }^{\circ} \mathrm{C}$ in $95 \%$ air-5\% $\mathrm{CO}_{2}$ (reperfusion). Cultures with sham treatment not deprived of oxygen and glucose were placed in BSS containing $20 \mathrm{mM}$ glucose.

\section{Measurement of extracellular glutamate}

Extracellular glutamate was measured using an enzymatic assay [31, 32]. In the presence of glutamate and $\beta$-nicotinamide adenine dinucleotide $\left(\mathrm{NAD}^{+}\right)$, L-glutamic dehydrogenase (GDH) produces $\alpha$-ketoglutarate and $\mathrm{NADH}$, a product that fluoresces when excited at 360 nm. In the presence of $\alpha$-ketoglutarate and L-Alanine, glutamate pyruvate transaminase (GPT) produces L-glutamate. Therefore, GDH (50 U/mL), NAD ${ }^{+}(2 \mathrm{mM})$, GPT (4 U/mL), and L-Alanine $(2 \mathrm{mM})$ were 
added to the external solution, and the fluorescence was excited at $360 \mathrm{~nm}$ and detected at $>510 \mathrm{~nm}$ with a fluorescence microscope. Images were acquired with integration times of $5 \mathrm{~s}$ at intervals of $20 \mathrm{~s}$. The extracellular glutamate was detected as an increase in NADH fluorescence.

\section{Western blot analysis}

Cell lysates were diluted 3:1 in sample buffer (187.5 mM Tris-HCl containing 6\% SDS, and 15\% glycerol, 15\% 2-mercaptoethanol) and denatured at $100{ }^{\circ} \mathrm{C}$ for 3 min. Proteins were electrophoresed on a $10 \%$ SDS-polyacrylamide gel and transferred onto a polyvinylidene difluoride membrane (Bio-Rad, Hercules, CA). Nonspecific binding sites were blocked with 5\% nonfat milk for $60 \mathrm{~min}$, and then the membrane was incubated overnight at $4{ }^{\circ} \mathrm{C}$ with the following antibodies: polyclonal antibody to GLT-1 (1:10000), and monoclonal antibody to actin (1:2000) (Sigma), and then horseradish peroxidase-labeled anti-guinea pig (1:2000) (Cell Signaling Technology) or anti- mouse IgG antibody for actin (1:2000) (Cell Signaling Technology), respectively. The immunoreactive 
bands were detected with an enhanced chemiluminescence kit (NEN Life Science Products, Boston, MA). Quantification of the levels was preformed by densitometric analysis using Scion Image Beta 4.02 (Scion Corporation).

\section{Survival rate of neurons}

Neuronal death was analyzed following observation of the nuclear morphology by using the fluorescent DNA-binding dyes, Hoechst 33342 (H33342) and propidium iodide (PI). Cells were incubated with these dyes for 15 minutes at $37{ }^{\circ} \mathrm{C}$. Individual nuclei were observed using a fluorescence microscope (Olympus, IX70) and subsequently analyzed. PI was used to identify nonviable cells. More specifically, an average of 450-500 neurons from fields chosen at random were analyzed in each experiment. The survival rate of neurons - meaning the percentage of viable neurons remaining — was determined by placing images of nuclear staining on phase-contrast images, and calculated as (viable neurons/total neurons before drug treatment) $\times 100$, since some neurons came off the dishes at the time of inspection. At least 4 independent experiments $(n \geq$ 
4) were conducted.

\section{Chemicals}

Forskolin, 2-Aminoethyl diphenylborinate, Pluronic F-127, NAD ${ }^{+}$, GDH, L-Alanine, GPT, Hoechst 33342 and propidium iodide were obtained from Sigma. Pituitary Adenylate Cyclase Activating Polypeptide 6-38 (Human) and Pituitary Adenylate Cyclase Activating Polypeptide 38 (Human) were obtained from Peptide Institute (Osaka, Japan). Fluo-4 AM was purchased from Molecular Probes. All other compounds were obtained from Wako Chemical (Tokyo, Japan).

\section{Statistics}

The data are expressed as the mean \pm S.D. Comparisons were made using the one-way analysis of variance (ANOVA) followed by a paired t-Test. A $P$ value of less than 0.01 or 0.05 was considered statistically significant. 


\section{RESULTS}

We investigated whether oscillations in the intracellular concentration of calcium ( $\mathrm{Ca}^{2+}$ oscillations) in neurons and/or astrocytes have any functional role in the development of preconditioning-induced neuronal ischemic tolerance in mixed cultures of neurons and astrocytes from fetal rat brain (embryonic day 17). Our recent studies have demonstrated that sublethal oxygen-glucose deprivation (OGD) for $60 \mathrm{~min}$ induces neuronal ischemic tolerance against subsequent lethal ODG for 90 min in neuron/astrocyte co-cultures [26, 27]. Neuronal ischemic tolerance was observed when the time between the PC and lethal OGD was $20-24 \mathrm{~h}$, but not when the interval was $6 \mathrm{~h}$ or $48 \mathrm{~h}$ [26]. Therefore, we first investigated whether the dynamics of $\mathrm{Ca}^{2+}$ oscillations changed during reperfusion $20 \mathrm{~h}$ after the end of PC.

Neurons and astrocytes were distinguished by GFAP staining after intracellular $\mathrm{Ca}^{2+}$ measurements (Figs. 1A2 and 1A3; see Materials and Methods). Spontaneous $\mathrm{Ca}^{2+}$ oscillations were observed in both neurons and astrocytes in our co-cultures (Fig. 1B1 and 1C1). About 50\% of the cultured neurons, and about $15 \%$ of cultured astrocytes located close to or 
under the aggregates of neurons, showed $\mathrm{Ca}^{2+}$ oscillations. At $4 \mathrm{~h}$ after the end of sublethal OGD (PC), the frequency of the spontaneous $\mathrm{Ca}^{2+}$ oscillations significantly decreased as compared with cells without PC (control) in both neurons and astrocytes (Fig. 1B2, 1B3, 1C2, and 1C3). The frequency of the oscillations then gradually increased, and had almost returned to the control level at $20 \mathrm{~h}$ after the PC in neurons and astrocytes (Fig. 1B3 and 1C3). The question then arises as to whether the transient decrease in the frequency of $\mathrm{Ca}^{2+}$ oscillations after the PC insult has anything to do with the development of the PC-induced ischemic tolerance of neurons.

We therefore investigated whether the forced suppression of spontaneous $\mathrm{Ca}^{2+}$ oscillations without the PC treatment before the onset of lethal OGD induced neuronal ischemic tolerance. Previous studies have suggested that the inositol 1, 4, 5-trisphosphate (IP3) receptors are involved in the generation of $\mathrm{Ca}^{2+}$ oscillations $[8,33]$, and the treatment of cells with 2-APB, an inhibitor of IP3 receptors, suppresses the oscillations [33]. The other chemicals that directly modulate the dynamics of intracellular $\mathrm{Ca}^{2+}$ oscillations, such as $\mathrm{Ca}^{2+}$ chelators and $\mathrm{Ca}^{2+}$ ionophore, 
would affect the basal level in intracellular $\mathrm{Ca}^{2+}$ concentration rather than the frequency of $\mathrm{Ca}^{2+}$ oscillations. We thus used 2-APB to suppress the spontaneous $\mathrm{Ca}^{2+}$ oscillations in our cultured cells. Treatment of cultures with 2-APB $(100 \mu \mathrm{M})$ didn't induce marked change in the base-line of the concentrations of intracellular free $\mathrm{Ca}^{2+}$, but significantly suppressed the spontaneous $\mathrm{Ca}^{2+}$ oscillations in both neurons and astrocytes in the cultures without PC (Fig. 2A and 2B). In addition, to clarify whether the 2-APB-induced suppression of $\mathrm{Ca}^{2+}$ oscillations contributed to the ischemic tolerance of neurons, the co-cultures were incubated in conditioned culture medium (CM) containing 2-APB (100 $\mu \mathrm{M})$ for $12 \mathrm{~h}$ before the onset of lethal OGD and then in CM without 2-APB for $8 \mathrm{~h}$ instead of being subjected to the PC treatment. Treatment with 2-APB significantly reduced neuronal cell death induced by lethal OGD (Fig. 2C). The survival rate of neurons with 2-APB treatment was almost the same as that with the prior PC insult.

We next investigated whether the inhibition of the PC-induced suppression of spontaneous $\mathrm{Ca}^{2+}$ oscillations reversed the PC-induced ischemic tolerance. A previous study [10] has suggested that treatment 
with forskolin, an activator of adenylate cyclase, increases the frequency of spontaneous $\mathrm{Ca}^{2+}$ oscillations. Although the frequency of spontaneous $\mathrm{Ca}^{2+}$ oscillations significantly decreased at $4 \mathrm{~h}$ after the end of the PC, treatment of cultures with forskolin $(50 \mu \mathrm{M})$ at $4 \mathrm{~h}$ after the end of the PC significantly increased the frequency oscillations in both neurons and astrocytes (Fig. 3A and 3B). In addition, we incubated the cultures in CM containing forskolin $(50 \mu \mathrm{M})$ for $12 \mathrm{~h}$ after the end of PC, and then in $\mathrm{CM}$ without the drug for $8 \mathrm{~h}$ after that. Treatment with forskolin significantly reversed the PC-induced ischemic tolerance of neurons (Fig. 3C). These results suggested that the increase in the oscillatory frequency before the onset of lethal OGD decreased the neuronal resistance to lethal OGD.

Our recent study [29] has demonstrated that the rise in the extracellular concentration of glutamate during OGD was mainly caused by a reversal in the operation of the astrocytic glutamate transporter GLT-1 in neuron/astrocyte co-cultures. In addition, the PC-induced transient decrease in the expression of astrocytic GLT-1 contributes both to the suppression of the rise in the extracellular concentration of glutamate 
during ischemia, and to the development of PC-induced ischemic tolerance. We therefore investigated whether the suppression of the spontaneous $\mathrm{Ca}^{2+}$ oscillations by 2-APB decreased the expression of astrocytic GLT-1 and the rise in the extracellular concentration of glutamate during ischemia. Figure 4A shows the time-course of the change in the extracellular concentration of glutamate during OGD in cultures treated with or without 2-APB $(100 \mu \mathrm{M})$ for $12 \mathrm{~h}$ before the onset of OGD and then in CM without 2-APB for $8 \mathrm{~h}$. OGD resulted in a marked rise in the concentration, but the elevation during OGD was significantly suppressed in co-cultures treated with 2-APB before the onset of lethal OGD (Fig. 4A and 4B). In addition, Western blotting showed that treatment with 2-APB for $12 \mathrm{~h}$ and then in CM without 2-APB for 8 h significantly reduced the expression level of GLT-1 (Fig. 4C and 4D), but did not reduce that of GLAST (Fig. 4E). These results suggested that the suppression of the spontaneous $\mathrm{Ca}^{2+}$ oscillations by 2-APB induced a down-regulation of GLT-1 expression contributing to PC-induced neuronal ischemic tolerance.

We next investigated possible factors that regulate the dynamics of 
spontaneous $\mathrm{Ca}^{2+}$ oscillations after the sublethal PC. Previous studies [34, 35] have suggested that pituitary adenylate cyclase activating polypeptide (PACAP), increases GLT-1 protein levels via the activation of PAC1 receptors in rat glial cultures. Our recent study [29] has demonstrated a possibility that PC insult suppresses the release of the neuron-derived factors up-regulating GLT-1 and that the down-regulation of GLT-1 contributes to the development of PC-induced ischemic tolerance. These finding have led to a hypothesis that PACAP would contribute to the regulation of astrocytic GLT-1 and the development of ischemic tolerance via the changes in the dynamics of $\mathrm{Ca}^{2+}$ oscillations regulating the expression of various genes. In addition, it has been suggested that both neurons and astrocytes express PAC1 receptors [36]. Therefore, there is a possibility that PACAP contributes to the regulation of spontaneous $\mathrm{Ca}^{2+}$ oscillations in both astrocytes and neurons. We next investigated whether PACAP was involved in the PC-induced changes in the dynamics of spontaneous $\mathrm{Ca}^{2+}$ oscillations and in the PC-induced neuronal ischemic tolerance. Treatment of co-cultures with PACAP6-38 (1 $\mu \mathrm{M})$, an inhibitor of PAC1 receptors [34], significantly suppressed the 
spontaneous $\mathrm{Ca}^{2+}$ oscillations in both neurons and astrocytes in the co-cultures without PC (Fig. 5A and 5B). Incubation of the cultures in conditioned medium (CM) containing PACAP6-38 $(1 \mu \mathrm{M})$ for $12 \mathrm{~h}$ before the onset of lethal OGD and then in CM without PACAP6-38 for $8 \mathrm{~h}$ resulted in a significant reduction in the neuronal cell death induced by lethal OGD (Fig. 5C), and the expression level of GLT-1 (Fig. 5D and 5E). In contrast, although the frequency of spontaneous $\mathrm{Ca}^{2+}$ oscillations significantly decreased at $4 \mathrm{~h}$ after the end of the PC, treatment of cultures with PACAP38 (100 nM) at $4 \mathrm{~h}$ after the sublethal PC insult significantly increased the frequency of spontaneous $\mathrm{Ca}^{2+}$ oscillations in the neurons and astrocytes (Fig. 6A and 6B). Incubation of the cultures in $\mathrm{CM}$ containing PACAP38 (100 nM) for $12 \mathrm{~h}$ after the end of PC and then in CM without PACAP38 for $8 \mathrm{~h}$ resulted in a significant decrease in the survival rate of neurons exposed to lethal OGD; that is, in the suppression of the PC-induced neuronal ischemic tolerance (Fig. 6C). In addition, the PC significantly lowered GLT-1 protein levels, but treatment with PACAP38 antagonized the PC-induced down-regulation of GLT-1 expression (Fig. 6D and 6E). 
Above findings suggested that treatment with PACAP38 antagonized PC-induced ischemic tolerance via PACAP-induced increase in $\mathrm{Ca}^{2+}$ oscillatory frequency. However, there remains a possibility that the PACAP-induced increase in $\mathrm{Ca}^{2+}$ oscillatory frequency does not directly contribute to the antagonization of PC-induced ischemic tolerance, since PACAP is known to be a factor activating a variety of intracellular signal transduction pathways. We finally investigated whether the attenuation of PC-induced ischemic tolerance by treatment with PACAP38 was recovered by co-treatment with 2-APB. Co-treatment of co-cultures with 2-APB $(100 \mu \mathrm{M})$ significantly suppressed the PACAP38 (100 nM) -induced increase in $\mathrm{Ca}^{2+}$ oscillatory frequency in both neurons and astrocytes (Fig. 7A and 7B). Incubation of the cultures in CM containing both PACAP38 $(100 \mathrm{nM})$ and 2-APB $(100 \mu \mathrm{M})$ for $12 \mathrm{~h}$ after the end of PC and then in CM without them for $8 \mathrm{~h}$ significantly reversed the PACAP38-induced reduction in the survival rate of neurons (Fig. 7C). These results supported our inference that the PACAP38-induced significant attenuation of the PC-induced ischemic tolerance was mainly caused by the PACAP38-induced increase in $\mathrm{Ca}^{2+}$ oscillatory frequency. 


\section{DISCUSSION}

This study showed that the frequency of spontaneous oscillations in the intracellular concentration of calcium ( $\mathrm{Ca}^{2+}$ oscillations) significantly decreased 4-8 $\mathrm{h}$ after the end of sublethal preconditioning (PC), and the decrease contributed to the development of ischemic tolerance in neurons 20-24 h after the PC. Previous studies have reported that the rise in the intracellular concentration of calcium was caused by a rise in the extracellular concentration of glutamate during PC, leading to the activation of calcium-dependent enzymes for the development of ischemic tolerance [22-24]. The present results, however, suggested that the changes in the dynamics of $\mathrm{Ca}^{2+}$ oscillations after the PC insult were crucial to the development of neuronal ischemic tolerance. In addition, here we provide experimental evidence suggesting that the changes in spontaneous $\mathrm{Ca}^{2+}$ oscillations in neurons and/or glias are involved in regulating the expression of some proteins, especially of astrocytic GLT-1 for the survival of neurons. Our recent study [29] and the other previous studies [37, 38] have reported that the decrease in the expression or the inhibition of astrocytic GLT-1 contributes to the suppression of the rise in 
the extracellular concentration of glutamate during ischemia, and to the protection to the ischemia-induced neuronal cell death. The present study suggested that the transient down-regulation of GLT-1 caused by the suppression of spontaneous $\mathrm{Ca}^{2+}$ oscillations after PC was one of the mechanisms for PC-induced ischemic tolerance.

The present study demonstrated that the frequency of spontaneous $\mathrm{Ca}^{2+}$ oscillations was suppressed at 4 and $8 \mathrm{~h}$ after the PC insult in both neurons and astrocytes, and the suppression contributed to the development of ischemic tolerance obtained $20 \mathrm{~h}$ after the PC. We also investigated the spontaneous calcium oscillations during the period 12 - 20 $\mathrm{h}$ after the PC. The frequency at $14 \mathrm{~h}$ after the PC almost recovered to the control level, but that at $18 \mathrm{~h}$ significantly increased (data not shown). However, the inhibition of the PC-induced increase in frequency at $18 \mathrm{~h}$ by 2-APB did not attenuate the PC-induced neuronal ischemic tolerance obtained at 20-24 h after the PC (data not shown). Although PC-induced ischemic tolerance is maintained for several hours in vitro and a few days in vivo, it disappears after that [13-15]. A previous study has demonstrated that the PC-induced tolerance in our co-cultures disappears 
at $48 \mathrm{~h}$ after the end of PC [26], and the PC-induced reduction in the level of astrocytic GLT-1 also recovers $48 \mathrm{~h}$ after the PC, suggesting a close relationship between the PC-induced change in GLT-1 expression and that in ischemic tolerance [29]. Therefore, the PC-induced rebound-like increase in the oscillatory frequency at $18 \mathrm{~h}$ after the PC may contribute to the recovery process, antagonizing the PC-induced temporal reduction in the expression of astrocytic GLT-1. In fact, the level of astrocytic GLT-1 had almost returned to that before the PC at $48 \mathrm{~h}$ after the insult [29]. In addition, the PC-induced ischemic tolerance was also attenuated significantly in association with the recovery of GLT-1 expression. The ischemic tolerance was observed at $20 \mathrm{~h}$ after the PC, when the PC-induced reduction in the frequency of $\mathrm{Ca}^{2+}$ oscillations was already reversed in both neurons and astrocytes (Fig. 1B3 and C3). There may be a considerable time delay linking the changes in the oscillatory frequency with those in the expression of proteins such as astrocytic GLT-1. This is probably why neuronal ischemic tolerance was still observed at $20 \mathrm{~h}$ after the PC.

The present study suggested that the PC-induced down-regulation of 
astrocytic GLT-1 and the resultant suppression in the rise in the concentration of extracellular glutamate during lethal ischemia were considered as the primary cause responsible for the development of PC-induced neuronal ischemic tolerance. However, there is a possibility that a sublethal PC would decrease the glutamate sensitivity in neurons, contributing to the PC-induced ischemic tolerance. Our previous study, however, has revealed that neurons rather become vulnerable to glutamate-mediated toxicity by the prior PC insult, probably caused by the PC-induced down-regulation of astrocytic GLT-1 leading to the reduction in the clearance capability of extracellular glutamate, suggesting that PC does not decrease the neuronal glutamate sensitivity at least in our co-cultures [29]. Taken together, our results suggested that the PC-induced down-regulation of astrocytic GLT-1 and the resultant suppression in the rise in the concentration of extracellular glutamate during lethal ischemia was critical to the development of PC-induced ischemic tolerance for neurons. However, under normal conditions, astrocytic GLT-1 plays a crucial role in the protection of neurons from glutamate-mediated toxicity. Therefore, it seems pathophysiologically 
meaningful for neurons that the PC-induced down-regulation of astrocytic GLT-1 expression is a transient phenomenon [29].

The present results demonstrated that the suppression of $\mathrm{Ca}^{2+}$ oscillations by treatment with either 2-APB (Fig. 4) or PACAP6-38 (Fig. 6) decreased the protein level of GLT-1. Then the question arises as to how $\mathrm{Ca}^{2+}$ oscillations participate regulating the expression of GLT-1. One possibility is the participation of a transcription factor, NF-кB. $\mathrm{NF}-\kappa \mathrm{B}$ is known to be one of the factors regulating the expression of GLT-1, and its activation leads to the up-regulation of GLT-1 expression [39, 40]. Dolmetsch et al. [7] reported that a decrease in $\mathrm{Ca}^{2+}$ oscillatory frequency reduced the gene expression induced by the activation of $\mathrm{NF}-\kappa \mathrm{B}$, suggesting that the decrease in oscillatory frequency results in the expression of astrocytic GLT-1 being down-regulated.

Our previous study has reported that the neuronal nitric oxide synthase (nNOS) derived NO produced during PC contributes to PC-induced down-regulation of GLT-1 and ischemic tolerance [41]. In the present study, there is no direct evidence that links NO production during PC and the suppression of $\mathrm{Ca}^{2+}$ oscillations after PC. However, 
some previous studies have reported the relationship between the regulation of $\mathrm{Ca}^{2+}$ oscillations or IP3 receptors and NO production. For example, the study in intact rat megakaryocytes reported that NO-cGMP inhibited $\mathrm{IP}_{3}$-induced $\mathrm{Ca}^{2+}$ release [42]. In addition, the study in neuronal nuclei of newborn piglets concluded that hypoxia resulted in increased functions for IP3 receptors by NO, and the modification of IP3 receptors may contribute to programmed cell death [43]. Thus, NO is involved in both physiological and pathological events, and is believed to play different roles in both lethal and sub-lethal events. Our present report demonstrated a possibility that NO produced during PC might affect the dynamics of $\mathrm{Ca}^{2+}$ oscillations after PC for PC-induced ischemic tolerance.

The protective role of astrocytic GLT-1 during ischemia is controversial. The operation of GLT-1 depends on the extracellular environment. Under the normal condition, astrocytic GLT-1 removes extracellular glutamate, and contributes to the protection against neurotoxic damage by glutamate. However under ischemic condition, astrocytic GLT-1 releases glutamate to extracellular space via the 
reverse-operation of GLT-1, and contributes to the induction of neurotoxic damage by glutamate. The studies about ischemia have been performed using in vitro [37, 44, 45] or in vivo [38, 46, 47] models, being different from the technique to induce ischemia, such as global or focal, and brain region, and these studies have reported inconsistent results [46, 47]. The changes in the extracellular environment during ischemia strongly depend on such ischemic models, and affect the function of glutamate transporters during ischemia. This point needs to be further investigated.

Previous studies have reported that several genes and thus proteins are involved in PC-induced neuronal ischemic tolerance. For some of the proteins, levels decrease [16, 17, 29], but for others, levels increase following the PC insult $[18,19]$. For example, the expression of Bcl-2, one of the anti-apoptosis proteins, increases on the activation of cyclic AMP-responsive element binding protein (CREB) after PC, and possibly contributes to the development of ischemic tolerance [18]. The present results couldn't explain all of the PC-induced changes in the expression of proteins. However, here we show that the PC-induced suppression of $\mathrm{Ca}^{2+}$ oscillations in neurons and/or astrocytes leading to a reduction in the 
expression of astrocytic GLT-1 was crucial to the development of PC-induced ischemic tolerance in neurons. Further study will be needed to clarify the precise mechanisms involved.

Previous studies have reported that PACAP induces neuroprotective effects through the phosphorylation of extracellular signal-regulated kinase (ERK); that is, mitogen-activated protein (MAP) kinase [48, 49, 50]. PACAP is also involved in the inhibition of Jun N-terminal kinase $(\mathrm{JNK}) /$ stress-activated protein kinase (SAPK) and p38, leading to anti-apoptotic effects under ischemic conditions [50]. The present study, however, demonstrated that in PACAP38-treated co-cultures, the PC-induced ischemic tolerance was significantly attenuated. In contrast, inhibition of the activity of PACAP receptors induced neuroprotective effects against OGD. Unlike previous reports, the present study indicated that neither the PACAP-induced activation of neuroprotective kinases such as a MAP kinase nor the PACAP-induced inhibition of apoptosis-inducing kinases such as SAPK had a primary role in the PC-induced increase in the ischemic tolerance of neurons.

Previous studies have reported that neuronal and astrocytic $\mathrm{Ca}^{2+}$ 
oscillations are regulated via extracellular messengers $[9,11]$. In fact, in our co-cultures, neurons and astrocytes showed synchronized $\mathrm{Ca}^{2+}$ oscillations in most of the experiments. It remains unclear whether the PC-induced changes in neuronal $\mathrm{Ca}^{2+}$ oscillations cause the changes in astrocytic oscillations or vice versa. We thus tried to clarify this issue using either PACAP, a neuron-derived peptide, which regulates the expression of astrocytic GLT-1, or an inhibitor of PACAP receptors. However, both PACAP38 and PACAP6-38 affected the calcium oscillations in neurons and in astrocytes. Not only neurons but also astrocytes have PACAP receptors [36]. In addition, the expression of PACAP has been confirmed in both neurons [51, 52] and cultured astrocytes although PACAP is expressed in astrocytes only from aged P0 rats [53]. Our previous paper, however, reported that the decrease in the release of PACAP from neurons is possibly involved in the PC-induced down-regulation of astrocytic GLT-1 expression [29]. A number of studies have also reported that the expression of astrocytic GLT-1 needs the existence of neurons $[34,54,55]$. Therefore, these results supported that the $\mathrm{Ca}^{2+}$ oscillation-regulated controlled release of PACAP from 
neurons rather than from astrocytes would play an essential role in the regulation of the expression of astrocytic GLT-1. More studies are needed to test this possibility. 


\section{Acknowledgements}

The authors wish to thank Mr. Hideomi Sato of the Research Institute

for Electronic Science, Hokkaido University, for help in establishing astrocyte/neuron co-cultures. 


\section{References}

1. Katz LC, Shatz CJ (1996) Synaptic activity and the construction of cortical circuits. Science 274:1133-1138

2. Komuro H, Rakic P (1998) Orchestration of neuronal migration by activity of ion channels, neurotransmitter receptors, and intracellular $\mathrm{Ca}^{2+}$ fluctuations. J Neurobiol 37:110-130

3. Feller MB (1999) Spontaneous correlated activity in developing neural circuits. Neuron 22:653-656

4. Spitzer NC, Lautermilch NJ, Smith RD, Gomez TM (2000) Coding of neuronal differentiation by calcium transients. Bioessays 22:811-817

5. Zhang LI, Poo MM (2001) Electrical activity and development of neural circuits. Nat Neurosci 4 suppl:1207-1214

6. Gu X, Spitzer NC (1995) Distinct aspects of neuronal differentiation encoded by frequency of spontaneous $\mathrm{Ca}^{2+}$ transients. Nature $375: 784-787$

7. Dolmetsch RE, Xu K, Lewis RS (1998) Calcium oscillations increase the efficiency and specificity of gene expression. Nature 392:933-936 
8. Li W, Llopis J, Whitney M, Zlokarnik G, Tsien RY (1998) Cell-permeant caged InsP3 ester shows that $\mathrm{Ca}^{2+}$ spike frequency can optimize gene expression. Nature 392:936-941

9. Pasti L, Volterra A, Pozzan T, Carmignoto G (1997) Intracellular calcium oscillations in astrocytes: a highly plastic, bidirectional form of communication between neurons and astrocytes in situ. J Neurosci $17: 7817-7830$

10.Gorbunova YV, Spitzer NC (2002) Dynamic interactions of cyclic AMP transients and spontaneous $\mathrm{Ca}^{2+}$ spikes. Nature 418:93-96

11.Morita M, Higuchi C, Moto T, Kozuka N, Susuki J, Itofusa R, Yamashita J, Kudo Y (2003) Dual regulation of calcium oscillation in astrocytes by growth factors and pro-inflammatory cytokines via the mitogen-activated protein kinase cascade. J Neurosci 23:10944-10952

12.Kitagawa K, Matsumoto M, Kuwabara K, Tagaya M, Ohtsuki T, Hata R, Ueda H, Handa N, Kimura K, Kamada T (1991) 'Ischemic tolerance' phenomenon detected in various brain regions. Brain Res 561:203-211

13.Kato H, Liu Y, Araki T, Kogure K (1992) MK-801, but not anisomycin, inhibits the induction of tolerance to ischemia in the gerbil 
hippocampus. Neurosci Lett 139:118-121

14.Bruer U, Weih MK, Isaev NK, Meisel A, Ruscher K, Bergk A, Trendelenburg G, Wiegand F, Victorov IV, Dirnagl U (1997) Induction of tolerance in rat cortical neurons: hypoxic preconditioning. FEBS Lett 414:117-121

15.Grabb MC, Choi DW (1999) Ischemic tolerance in murine cortical cell culture: critical role for NMDA receptors. J Neurosci 19:1657-1662

16.Douen AG, Akiyama K, Hogan MJ, Wang F, Dong L, Chow AK, Hakim A (2000) Preconditioning with cortical spreading depression decreases intraischemic cerebral glutamate levels and down-regulates excitatory amino acid transporters EAAT1 and EAAT2 from rat cerebal cortex plasma membranes. J Neurochem 75:812-818

17.Katsura KI, Kurihara J, Kato H, Katayama Y (2001) Ischemic pre-conditioning affects the subcellular distribution of protein kinase $\mathrm{C}$ and calcium/calmodulin-dependent protein kinase II in the gerbil hippocampal CA1 neurons. Neurol Res 23:751-754

18.Wu C, Fujihara H, Yao J, Qi S, Li H, Shimoji K, Baba H (2003) Different expression patterns of Bcl-2, Bcl-xl, and Bax proteins after 
sublethal forebrain ischemia in C57Black/Crj6 mouse striatum. Stroke 34:1803-1808

19.Naylor M, Bowen KK, Sailor KA, Dempsey RJ, Vemuganti R (2005) Preconditioning-induced ischemic tolerance stimulates growth factor expression and neurogenesis in adult rat hippocampus. Neurochem Int $47: 565-572$

20.Nakamura T, Minamisawa H, Katayama Y, Ueda M, Terashi A, Nakamura K, Kudo Y (1999) Increased intracellular $\mathrm{Ca}^{2+}$ concentration in the hippocampal CA1 area during global ischemia and reperfusion in the rat: a possible cause of delayed neuronal death. Neuroscience 88:57-67

21.Martinez-Sanchez M, Striggow F, Schroder UH, Kahlert S, Reymann KG, Reiser G (2004) $\mathrm{Na}^{+}$and $\mathrm{Ca}^{2+}$ homeostasis pathways, cell death and protection after oxygen-glucose-deprivation in organotypic hippocampal slice cultures. Neuroscience 128:729-740

22.Gidday JM, Shah AR, Maceren RG, Wang Q, Pelligrino DA, Holtzman DM, Park TS (1999) Nitric oxide mediates cerebral ischemic tolerance in a neonatal rat model of hypoxic preconditioning. J Cereb Blood 
Flow Metab 19:331-340

23.Mabuchi T, Kitagawa K, Kuwabara K, Takasawa K, Ohtsuki T, Xia Z, Storm D, Yanagihara T, Hori M, Matsumoto M (2001) Phosphorylation of cAMP response element-binding protein in hippocampal neurons as a protective response after exposure to glutamate in vitro and ischemia in vivo. J Neurosci 21:9204-9213

24.Tauskela JS, Morley P (2004) On the role of $\mathrm{Ca}^{2+}$ in cerebral ischemic preconditioning. Cell Calcium 36:313-322

25.Kawahara K, Hosoya R, Sato H, Tanaka M, Nakajima T, Iwabuchi S (2002) Selective blockade of astrocytic glutamate transporter GLT-1 with dihydrokainate prevents neuronal death during ouabain treatment of astrocyte/neuron cocultures. Glia 40:337-349

26.Kawahara K, Yanoma J, Tanaka M, Nakajima T, Kosugi T (2004) Nitric oxide produced during ischemia is toxic but crucial to preconditioning-induced ischemic tolerance of neurons in culture. Neurochem Res 29:797-804

27.Kawahara K, Kosugi T, Tanaka M, Nakajima T, Yamada T (2005) Reversed operation of glutamate transporter GLT-1 is crucial to the 
development of preconditioning-induced ischemic tolerance of neurons in neuron/astrocyte co-cultures. Glia 49:349-359

28.Conde Guerri B, Sinues Porta E, Arrazola Schlamilch M, Comunas Gonzalez F, Calatayud Maldonado V (1989) Effects of glia-conditioned medium on primary cultures of central neurons. Histol Histopathol 4: $217-222$

29.Kosugi T, Kawahara K, Yamada T, Nakajima T, Tanaka M (2005) Functional significance of the preconditioning-induced down-regulation of glutamate transpoter GLT-1 in neuron/astrocyte co-cultures. Neurochem Res 30:1109-1116

30.Yamada T, Kawahara K, Kosugi T, Tanaka M (2006) Nitric oxide produced during sublethal ischemia is crucial for the preconditioning-induced down-regulation of glutamate transporter GLT-1 in neuron/astrocyte co-cultures. Neurochem Res 31:49-56

31.Maguire G, Simko H, Weinreb RN, Ayoub G (1998) Transport-mediated release of endogenous glutamate in the vertebrate retina. Pflugers Arch 436:481-484

32.Innocenti B, Parpura V, Haydon PG (2000) Imaging extracellular 
waves of glutamate during calcium signaling in cultured astrocytes. $\mathrm{J}$ Neurosci 20:1800-1808

33.Parri HR, Crunelli V (2003) The role of $\mathrm{Ca}^{2+}$ in the generation of spontaneous astrocytic $\mathrm{Ca}^{2+}$ oscillations. Neuroscience 120:979-992

34.Figiel M, Engele J (2000) Pituitary adenylate cyclase-activating polypeptide (PACAP), a neuron-derived peptide regulating glial glutamate transport and metabolism. J Neurosci 20:3596-3605

35.Schluter K, Figiel M, Rozyczka J, Engele J (2002) CNS region-specific regulation of glial glutamate transporter expression. Eur J Neurosci $16: 836-842$

36.Joo KM, Chung YH, Kim MK, Nam RH, Lee BL, Lee KH, Cha CI (2004) Distribution of vasoactive intestinal peptide and pituitary adenylate cyclase-activating polypeptide receptors (VPAC1, VPAC2, and PAC1 receptor) in the rat brain. J Comp Neurol 476:388-413

37.Phillis JW, Smith-Barbour M, Perkins LM, O'Regan MH. (1994) Characterization of glutamate, aspartate, and GABA release from ischemic rat cerebral cortex. Brain Res Bull. 34:457-66

38.Seki Y, Feustel PJ, Keller RW Jr, Tranmer BI, Kimelberg HK. (1999) 
Inhibition of ischemia-induced glutamate release in rat striatum by dihydrokinate and an anion channel blocker. Stroke. 30:433-40

39.Zelenaia O, Schlag BD, Gochenauer GE, Ganel R, Song W, Beesley JS, Grinspan JB, Rothstein JD, Robinson MB (2000) Epidermal growth factor receptor agonists increase expression of glutamate transporter GLT-1 in astrocytes through pathways dependent on phosphatidylinositol 3-kinase and transcription factor NF-kappaB. Mol Pharmacol 57:667-678

40.Rodriguez-Kern A, Gegelashvili M, Schousboe A, Zhang J, Sung L, Gegelashvili G (2003) Beta-amyloid and brain-derived neurotrophic factor, BDNF, up-regulate the expression of glutamate transporter GLT-1/EAAT2 via different signaling pathways utilizing transcription factor NF-kappaB. Neurochem Int 43:363-370

41.Yamada T, Kawahara K, Kosugi T, Tanaka M. (2006) Nitric oxide produced during sublethal ischemia is crucial for the preconditioning-induced down-regulation of glutamate transporter GLT-1 in neuron/astrocyte co-cultures. Neurochem Res. 31:49-56

42.Tertyshnikova S, Yan X, Fein A. (1998) cGMP inhibits IP3-induced 
$\mathrm{Ca}^{2+}$ release in intact rat megakaryocytes via cGMP- and cAMP-dependent protein kinases. J Physiol. 512:89-96

43.Mishra OP, Qayyum I, Delivoria-Papadopoulos M. (2003) Hypoxia-induced modification of the inositol triphosphate receptor in neuronal nuclei of newborn piglets: role of nitric oxide. J Neurosci Res. 74:333-8

44.Rossi DJ, Oshima T, Attwell D. (2000) Glutamate release in severe brain ischaemia is mainly by reversed uptake. Nature. 403:316-21

45.Nelson RM, Lambert DG, Richard Green A, Hainsworth AH. (2003) Pharmacology of ischemia-induced glutamate efflux from rat cerebral cortex in vitro. Brain Res. 964:1-8

46. Rao VL, Dogan A, Todd KG, Bowen KK, Kim BT, Rothstein JD, Dempsey RJ. (2001) Antisense knockdown of the glial glutamate transporter GLT-1, but not the neuronal glutamate transporter EAAC1, exacerbates transient focal cerebral ischemia-induced neuronal damage in rat brain. J Neurosci. 21:1876-83

47.Mitani A, Tanaka K. (2003) Functional changes of glial glutamate transporter GLT-1 during ischemia: an in vivo study in the hippocampal 
CA1 of normal mice and mutant mice lacking GLT-1. J Neurosci. 23:7176-82

48. Vaudry D, Gonzalez BJ, Basille M, Anouar Y, Fournier A, Vaudry H. (1998) Pituitary adenylate cyclase-activating polypeptide stimulates both c-fos gene expression and cell survival in rat cerebellar granule neurons through activation of the protein kinase A pathway. Neuroscience 84:801-812

49.Vaudry D, Pamantung TF, Basille M, Rousselle C, Fournier A, Vaudry H, Beauvillain JC, Gonzalez BJ (2002) PACAP protects cerebellar granule neurons against oxidative stress-induced apoptosis. Eur $\mathrm{J}$ Neurosci 15:1451-1460

50.Dohi K, Mizushima H, Nakajo S, Ohtaki H, Matsunaga S, Aruga T, Shioda S (2002) Pituitary adenylate cyclase-activating polypeptide (PACAP) prevents hippocampal neurons from apoptosis by inhibiting JNK/SAPK and p38 signal transduction pathways. Regul Pept 109:83-88

51.Arimura A (1992) Pituitary adenylate cyclase activating polypeptide (PACAP): discovery and current status of research. Regul Pept 
$37: 287-303$

52.Shintani N, Suetake S, Hashimoto H, Koga K, Kasai A, Kawaguchi C, Morita Y, Hirose M, Sakai Y, Tomimoto S, Matsuda T, Baba A (2005) Neuroprotective action of endogenous PACAP in cultured rat cortical neurons. Regul Pept 126:123-128

53.Jaworski DM (2000) Expression of pituitary adenylate cyclase-activating polypeptide (PACAP) and the PACAP-selective receptor in cultured rat astrocytes, human brain tumors, and in response to acute intracranial injury. Cell Tissue Res 300:219-230

54.Gegelashvili G, Dehnes Y, Danbolt NC, Schousboe A (2000) The high-affinity glutamate transporters GLT1, GLAST, and EAAT4 are regulated via different signalling mechanisms. Neurochem Int $37: 163-170$

55.Poitry-Yamate CL, Vutskits L, Rauen T (2002) Neuronal-induced and glutamate- dependent activation of glial glutamate transporter function. J Neurochem 82:987-997 


\section{Figure legends}

Fig.1.

Changes in the spontaneous calcium oscillations in neurons and astrocytes after the end of the preconditioning (PC) insult. Figures A1, A2, and A3 show a representative photomicrograph, a fluorescent image using Fluo4, and the fluorescent labeling of GFAP in the same region of a co-culture, respectively. B1-B3: Representative spontaneous calcium oscillations observed in a neuron in the cultures without PC (control, Cont.) (B1), at $4 \mathrm{~h}$ after the PC (B2), and a bar graph showing the average frequency of calcium oscillations of neurons for 15 min (B3). C1-C3: Representative spontaneous calcium oscillations in an astrocyte in the non-PC cultures (control, Cont.) (C1), at $4 \mathrm{~h}$ after the PC (C2), and a bar graph showing the average frequency of calcium oscillations of astrocytes for 15 min (C3). Data are expressed as the mean $+\mathrm{SD}(n=4$, different cultures). $\quad * P<0.05$. 


\section{Fig.2.}

Treatment with 2-APB suppressed the spontaneous calcium oscillations, and induced neuronal ischemic tolerance. A: Representative spontaneous calcium oscillations in a neuron with (A2) or without (A1) 2-APB (100 $\mu \mathrm{M}$ ) treatment in the cultures without PC, and in an astrocyte with (A4) or without (A3) 2-APB treatment in the cultures without PC. B: Average frequency of calcium oscillations in neurons (B1) or astrocytes (B2) in the control (Cont.) and on treatment with 2-APB for $10 \mathrm{~min}$. C: Statistical comparison of the survival rate of neurons following lethal OGD. Data are expressed as the mean $+\mathrm{SD}(\mathrm{n}=4 \sim 5$, different cultures). $\quad * P<0.05$

Fig.3.

Treatment with forskolin enhanced the spontaneous calcium oscillations and reversed the PC-induced neuronal ischemic tolerance. A: Representative spontaneous calcium oscillations in a neuron with (A2) or without (A1) forskolin (50 $\mu \mathrm{M})$ treatment at $4 \mathrm{~h}$ after the end of PC, and in an astrocyte with (A4) or without (A3) forskolin treatment at $4 \mathrm{~h}$ after the end of PC. B: Average frequency of the calcium oscillations in neurons 
(B1) or astrocytes (B2) in the cultures at $4 \mathrm{~h}$ after the end of the PC (PC 4h), and in the cultures treated with forskolin for $10 \mathrm{~min}$ at $4 \mathrm{~h}$ after the end of the PC,. C: Statistical comparison of the survival rate of neurons following lethal OGD. Data are expressed as the mean $+\mathrm{SD}(n=4$, different cultures). $\quad * P<0.05$.

Fig.4.

Treatment with 2-APB reduced the rise in the extracellular concentration of glutamate during OGD and the expression of GLT-1. A: Time course of the change in the NADH fluorescence ratio during OGD in control cultures (triangle), and in the cultures incubated in CM for $8 \mathrm{~h}$ following the treatment with 2-APB $(100 \mu \mathrm{M})$ for $12 \mathrm{~h}$ before the onset of OGD (square), respectively. Control (Cont.): cultures treated with $0.1 \%$ DMSO, a vehicle of 2-APB. OGD begins at $0 \mathrm{~min}$. Vertical bars indicate either + or - SD. B: Statistical comparison of the relative fluorescence intensity 80 min after the onset of OGD. Data are expressed as the mean $+\mathrm{SD}\left(\mathrm{n}=4-7\right.$ different cultures). ${ }^{*} P<0.05 . \quad \mathrm{C}:$ GLT-1, GLAST and actin protein expression levels analyzed by Western blotting in the control 
cultures or the cultures incubated in $\mathrm{CM}$ for $8 \mathrm{~h}$ following the treatment with 2-APB for $12 \mathrm{~h}$. D and E: Statistical comparison of the expression of GLT-1 and GLAST normalized to that of actin. Data are expressed as the mean $+\mathrm{SD}$ ( $\mathrm{n}=4$ different cultures). ${ }^{*} \mathrm{P}<0.05$.

Fig.5.

Treatment with PACAP 6-38 suppressed the spontaneous calcium oscillations, and induced neuronal ischemic tolerance. A: Representative spontaneous calcium oscillations in a neuron with (A2) or without (A1) PACAP 6-38 $(1 \mu \mathrm{M})$ treatment in the cultures without PC, and in an astrocyte with (A4) or without (A3) PACAP 6-38 treatment in the cultures without PC. B: Average frequency of calcium oscillations in neurons (B1) or astrocytes (B2) in the control cultures (Cont.) and in the cultures treated with PACAP 6-38 for 10 min. C: Statistical comparison of the survival rate of neurons following lethal OGD. D: Expression of GLT-1 and actin analyzed by Western blotting in the cultures incubated in CM for $8 \mathrm{~h}$ following the treatment with or without PACAP 6-38 for $12 \mathrm{~h}$. E: Statistical comparison of the expression of GLT-1 normalized to the 
expression of actin. Data are expressed as the mean $+S D(n=4$, different cultures). $\quad * P<0.05$

Fig.6.

Treatment with PACAP 38 enhanced the spontaneous calcium oscillations, and reversed the PC-induced neuronal ischemic tolerance. A: Representative spontaneous calcium oscillations in a neuron with (A2) or without (A1) PACAP $38(100 \mathrm{nM})$ treatment at $4 \mathrm{~h}$ after the end of PC, and in an astrocyte with (A4) or without (A3) PACAP 38 treatment at $4 \mathrm{~h}$ after the end of PC. B: Average frequency of calcium oscillations in neurons (B1) or astrocytes (B2) in the cultures at $4 \mathrm{~h}$ after the end of the PC (PC 4h), and in the cultures treated with PACAP 38 for 10 min at $4 \mathrm{~h}$ after the end of the PC,. C: Statistical comparison of the survival rate of neurons following lethal OGD. D: Expression of GLT-1 and actin analyzed by Western blotting in the control cultures (sham-treated) and in the cultures incubated in $\mathrm{CM}$ for $8 \mathrm{~h}$ following treatment with or without PACAP38 for $12 \mathrm{~h}$ after the end of PC. E: Statistical comparison of the expression of GLT-1 normalized to the expression of actin. Data are 
expressed as the mean $+\mathrm{SD}(\mathrm{n}=4$, different cultures). $\quad * P<0.05$.

\section{Fig.7.}

Co-treatment with 2-APB antagonized both the PACAP-induced increase in the spontaneous calcium oscillatory frequency and the PACAP-induced attenuation of the PC-induced neuronal ischemic tolerance. A: Figures A1-A3 show representative spontaneous calcium oscillations in a neuron co-treated with PACAP 38 (100 nM) and 2-APB (100 $\mu \mathrm{M})(\mathrm{A} 3)$, treated with PACAP 38 only (A2), and control (A1) at $4 \mathrm{~h}$ after the end of PC, respectively. Figures A4-A6 show calcium oscillations in an astrocyte co-treated with PACAP $38(100 \mathrm{nM})$ and 2-APB $(100 \mu \mathrm{M})(\mathrm{A} 6)$, treated with PACAP 38 only (A5), and control (A4) at $4 \mathrm{~h}$ after the end of PC, respectively. B: Average frequency of calcium oscillations in neurons (B1) or astrocytes (B2) in the cultures at 4 $\mathrm{h}$ after the end of the PC (PC 4h), and in the cultures treated with PACAP 38 or co-treated with PACAP 38 and 2-APB for $10 \mathrm{~min}$ at $4 \mathrm{~h}$ after the end of the PC. C: Statistical comparison of the survival rate of neurons following lethal OGD. 


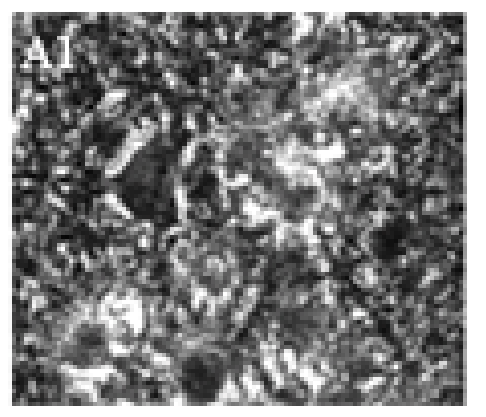

Nemon

B1

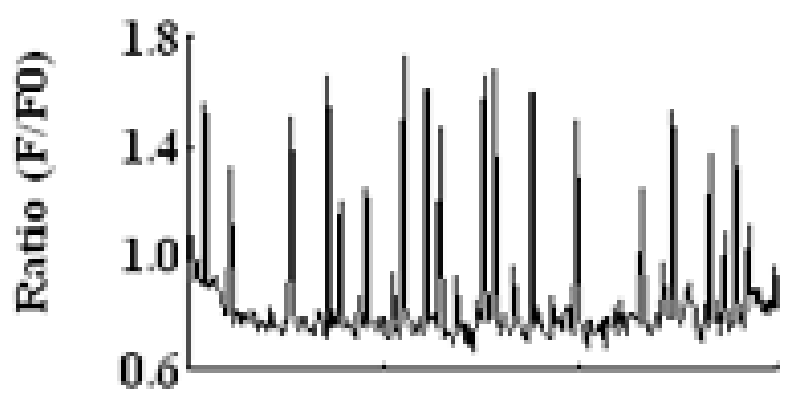

B2
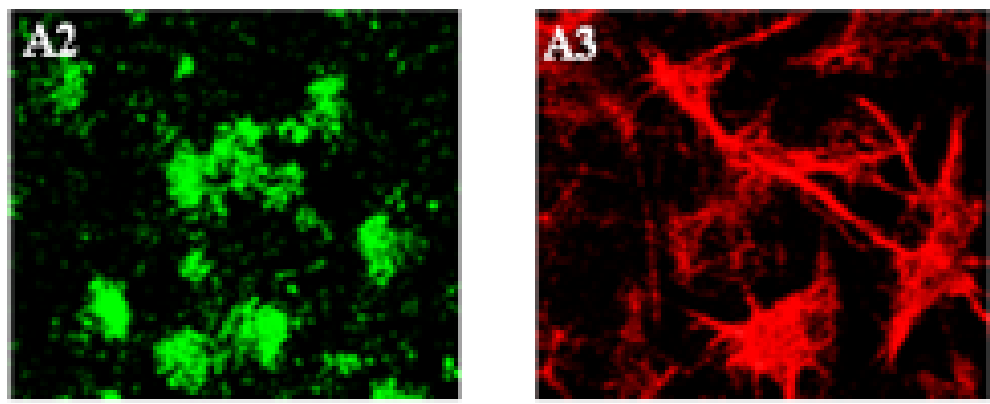

Astrocyte

C1

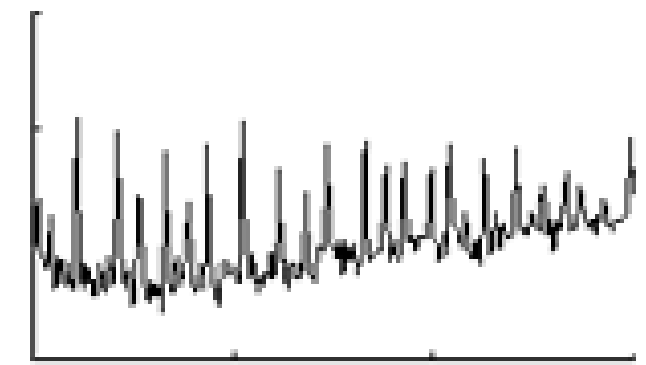

C2

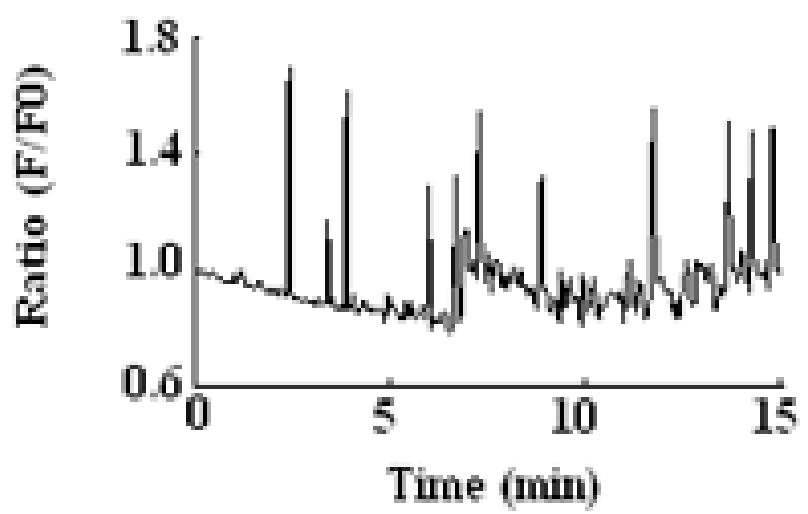

B3

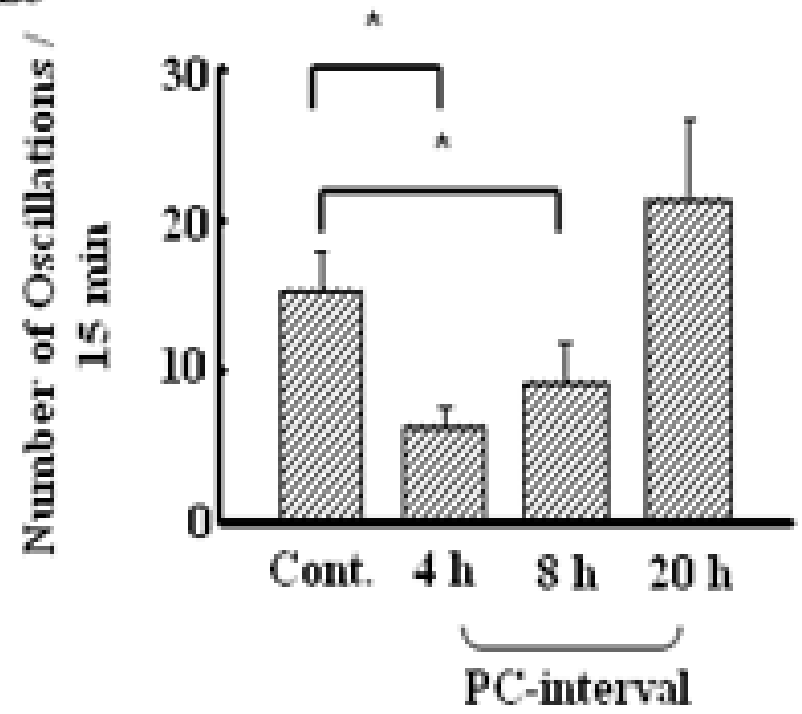

C3

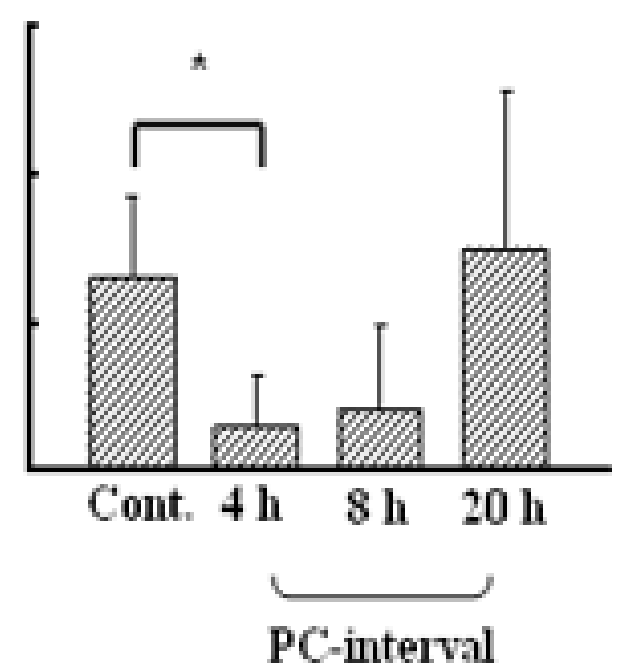


Newron

Al

A2

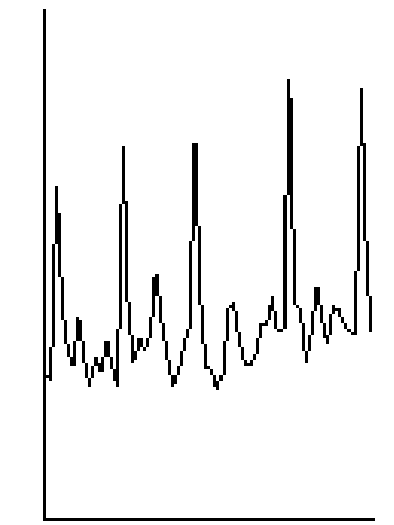

Astrocyte

A3

A4

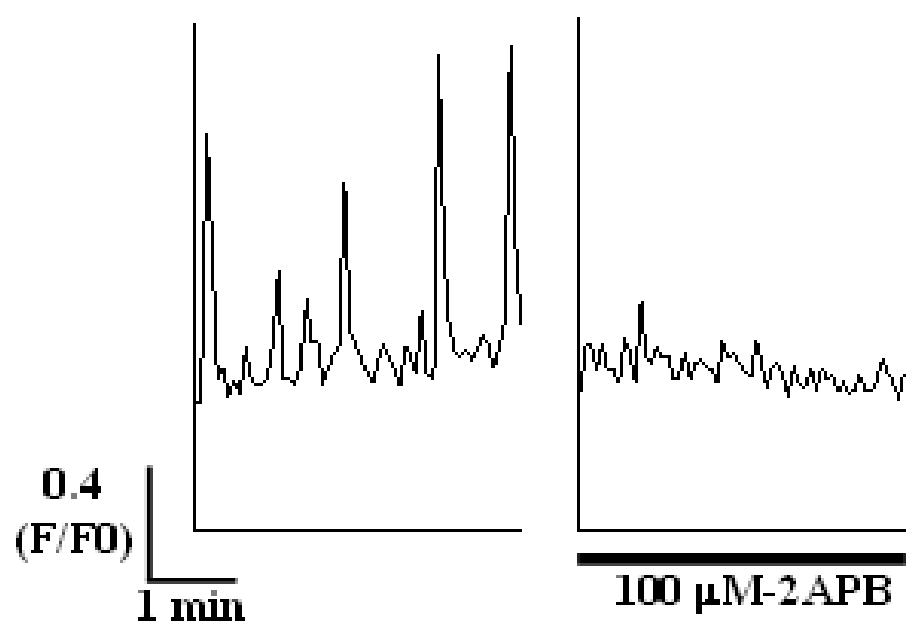

C

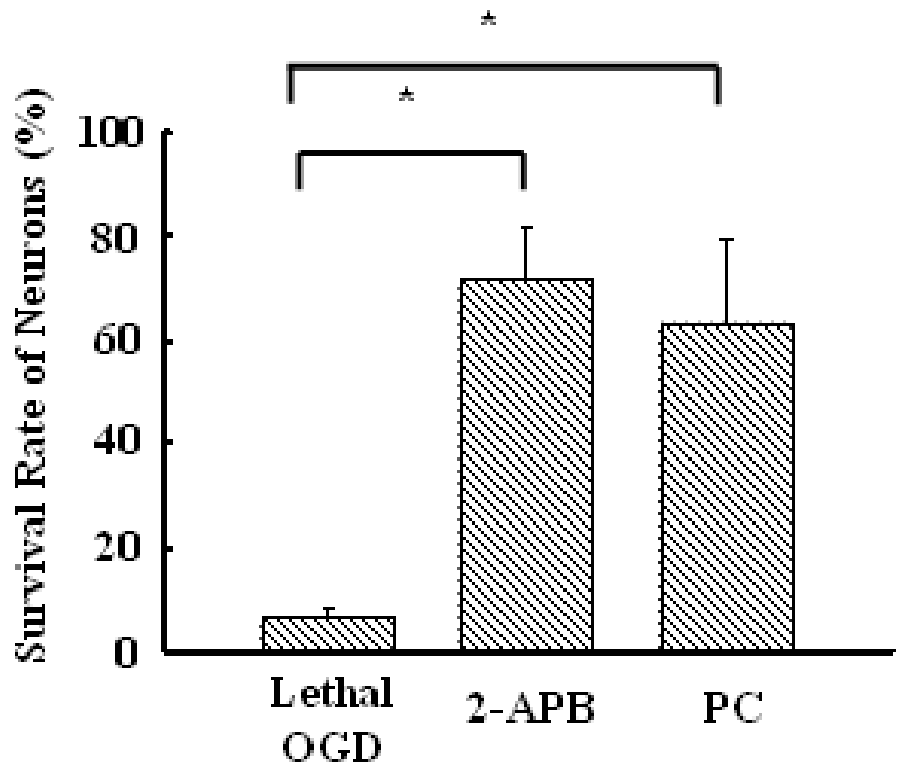

B1

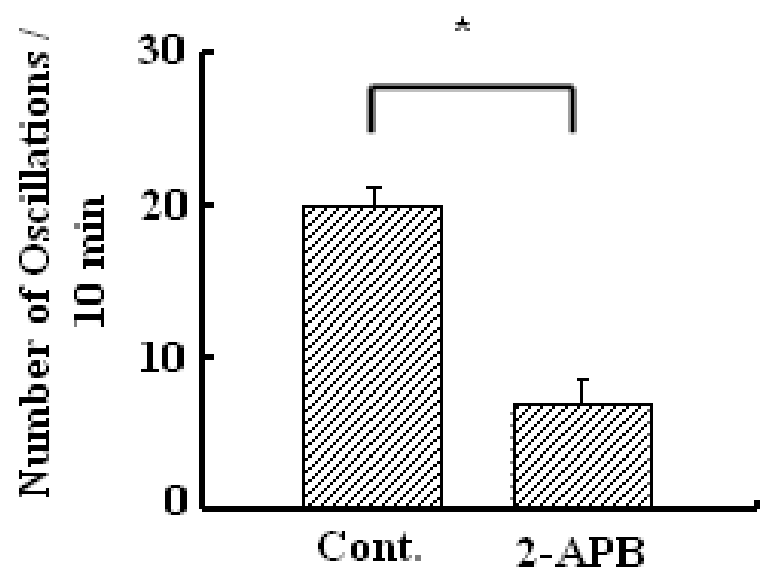

B2

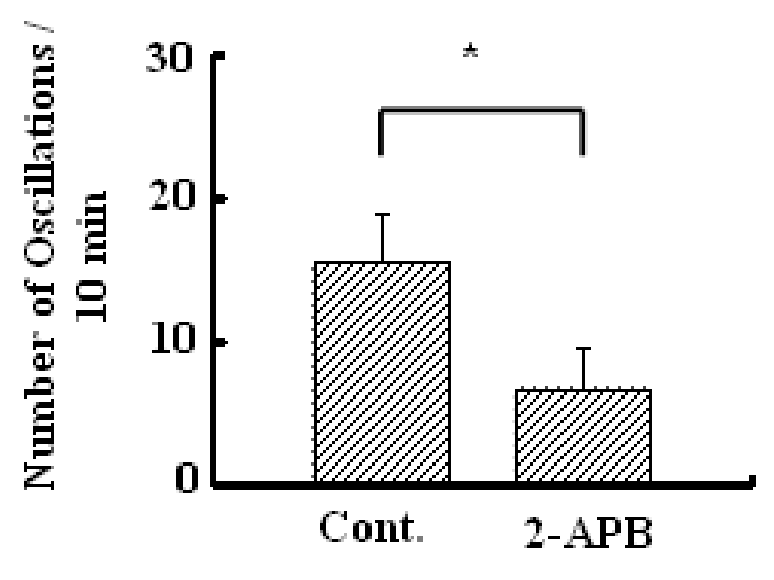


Neuron

Al

A2

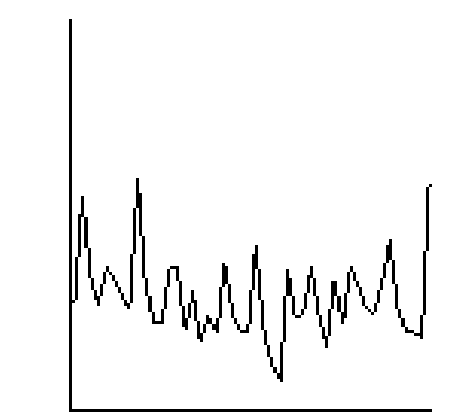

Astrocyte
N

B1

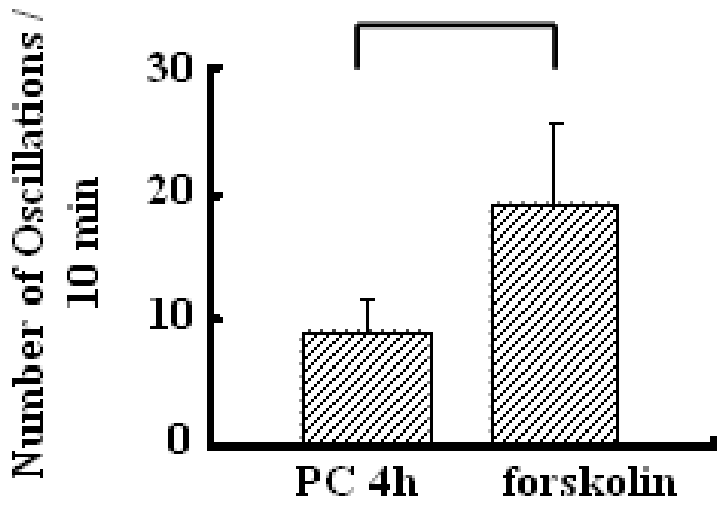

A3

A4

B2

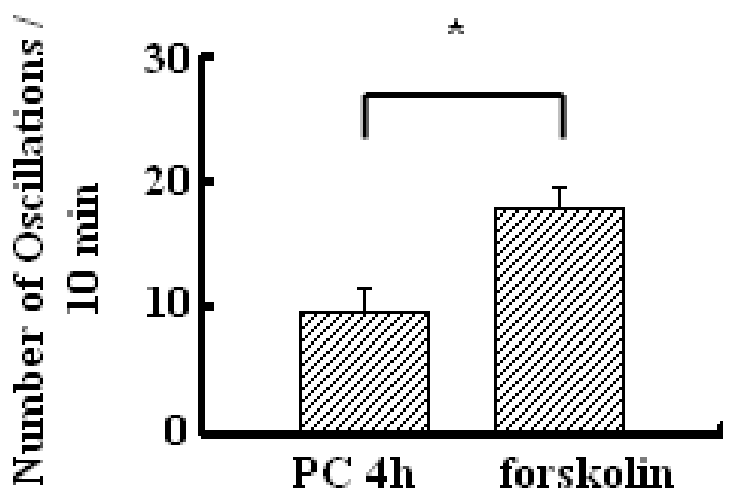

(F/FO)

1 min

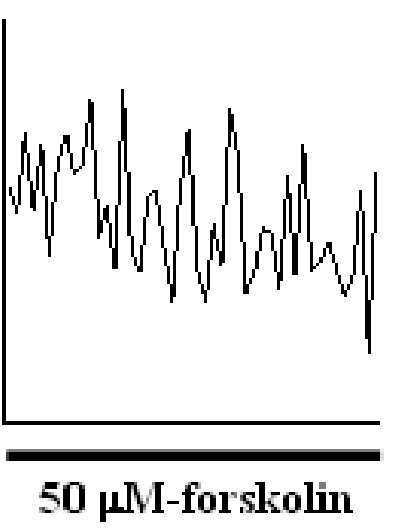

$\mathrm{C}$

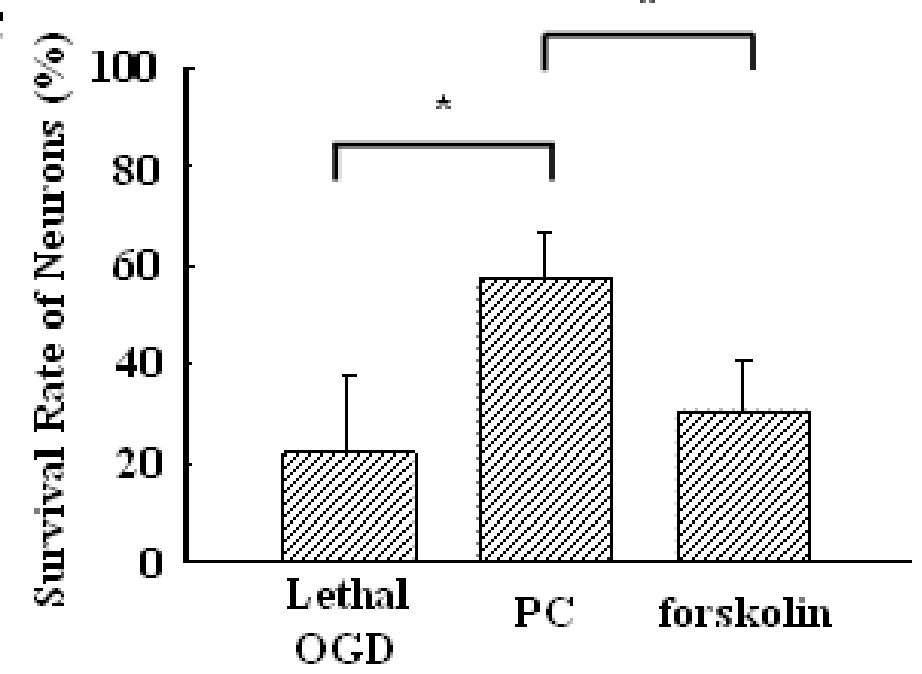




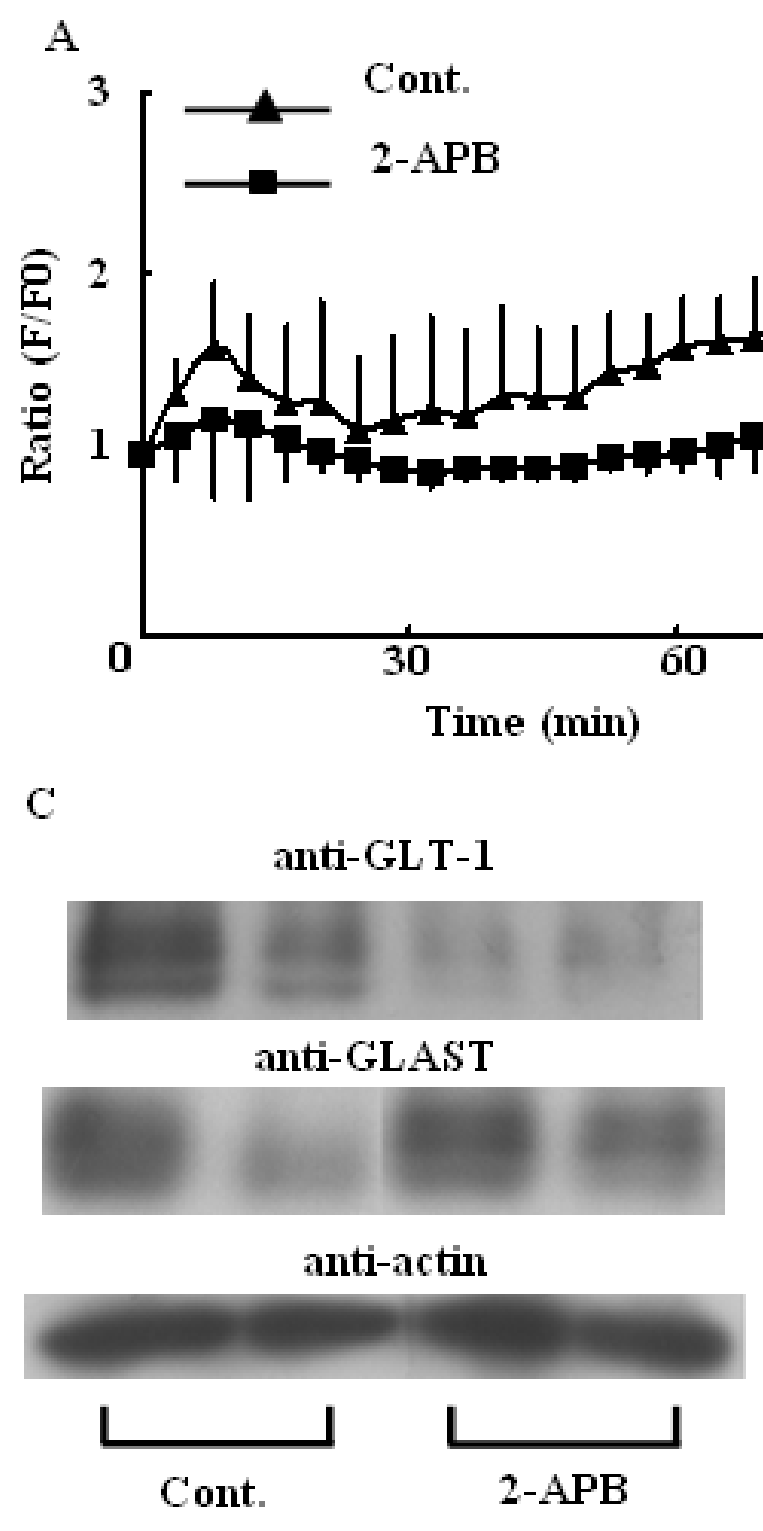

B

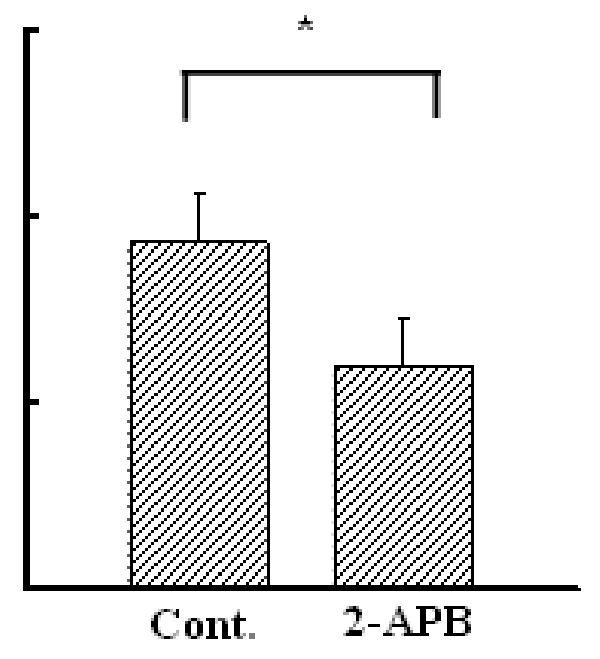

D

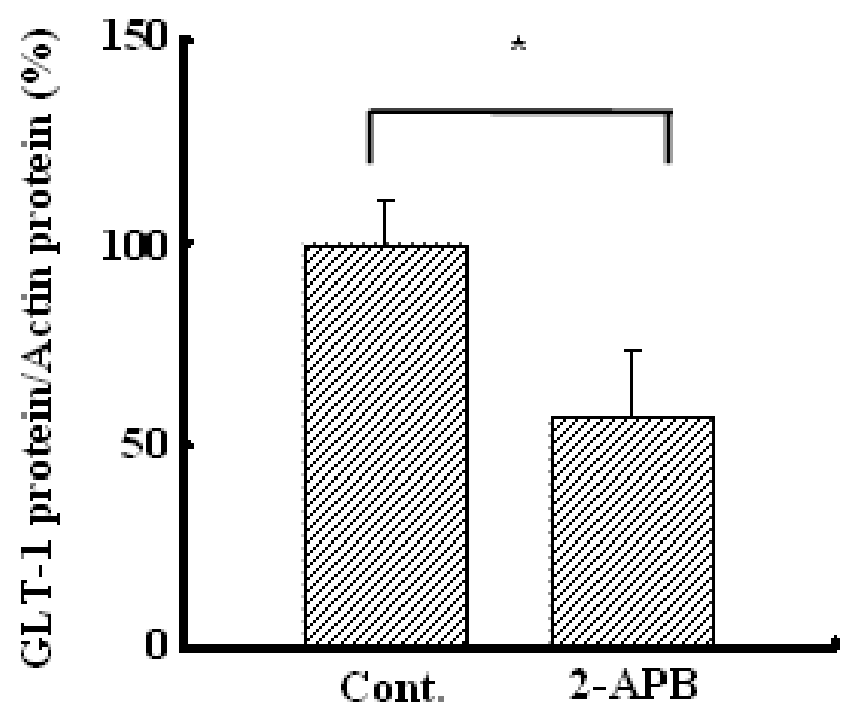

E

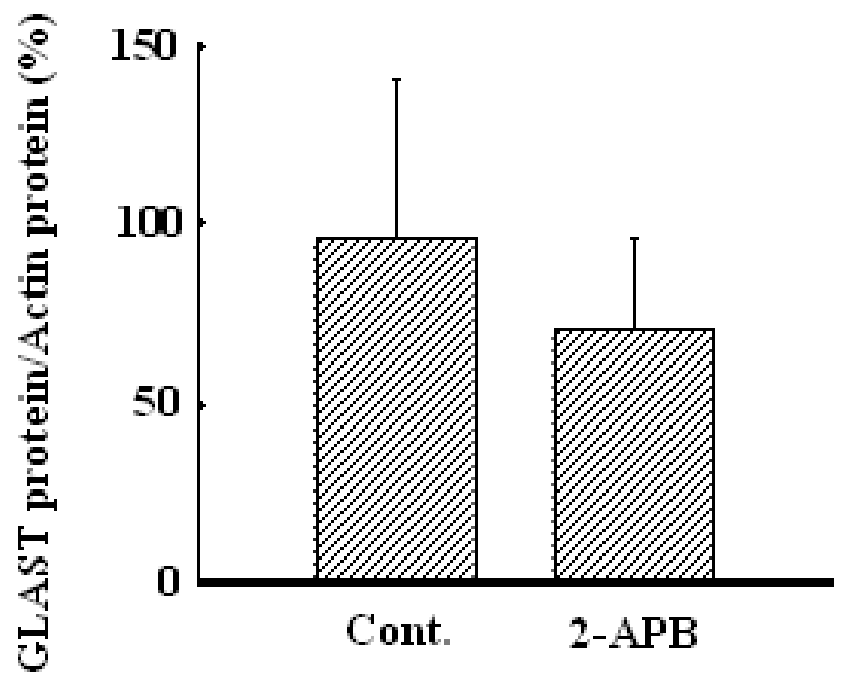


Neuron

Al
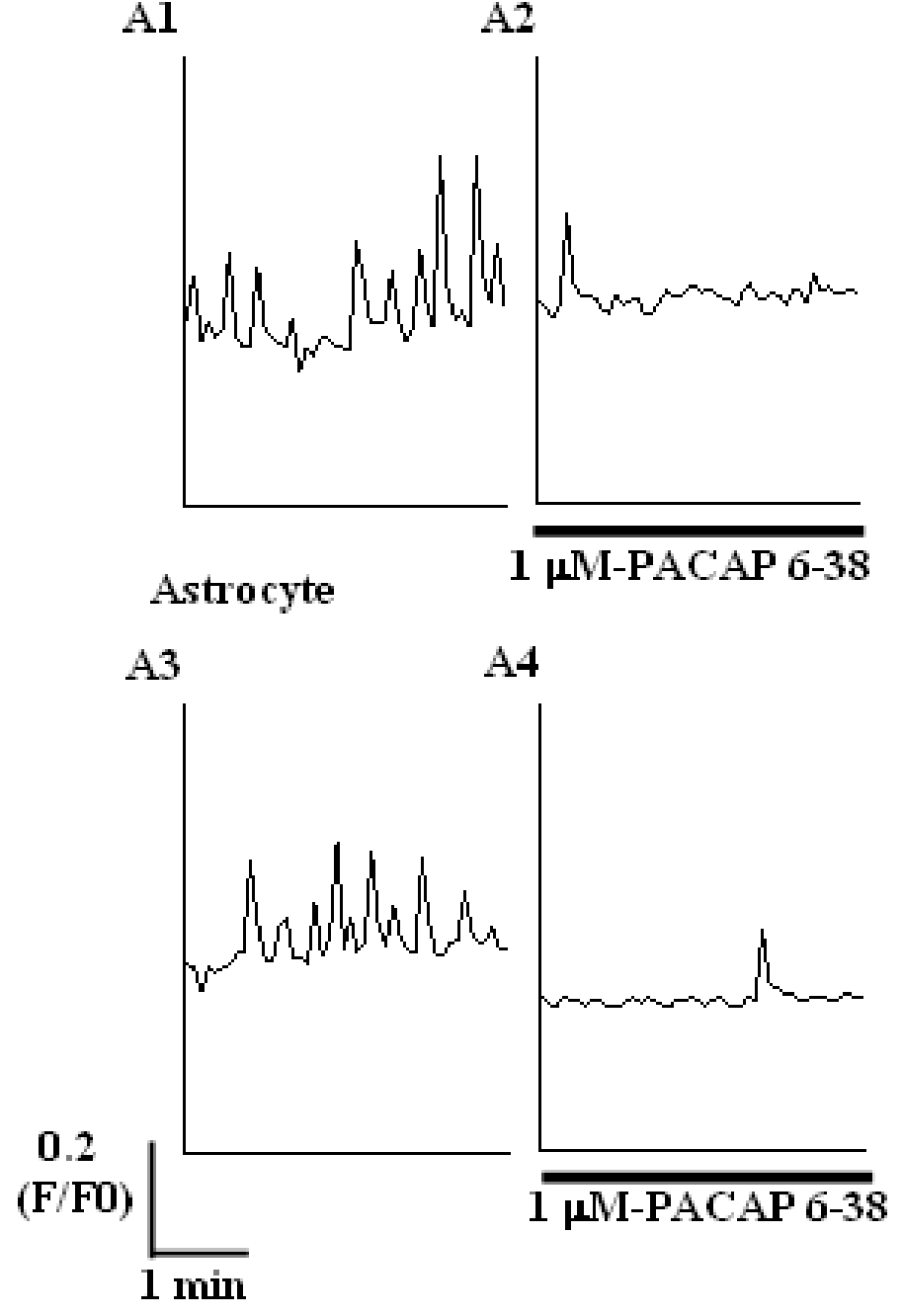

C

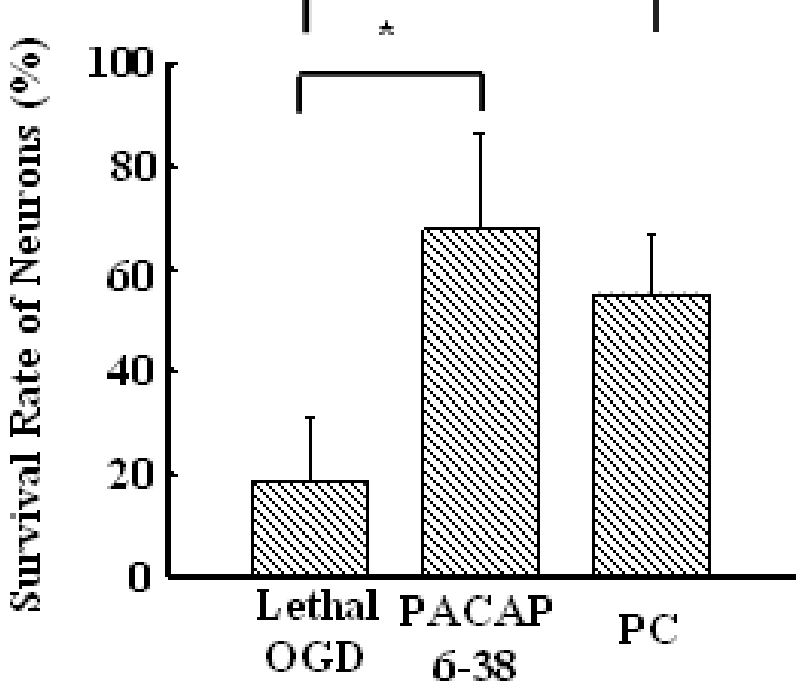

B1

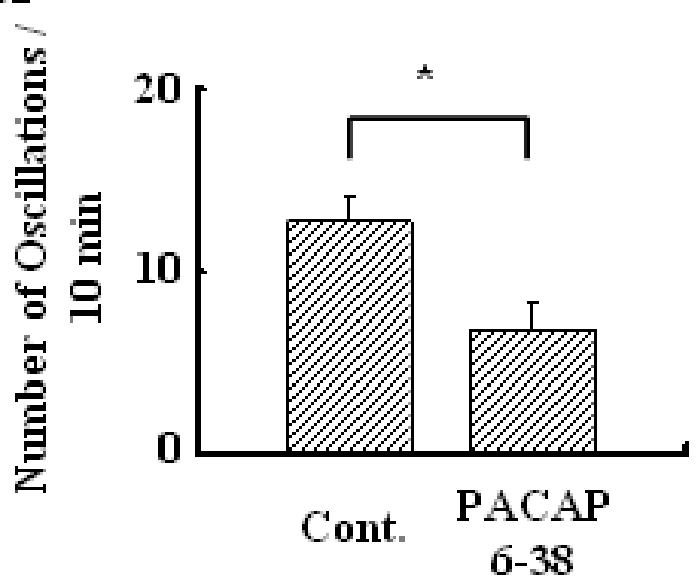

B2

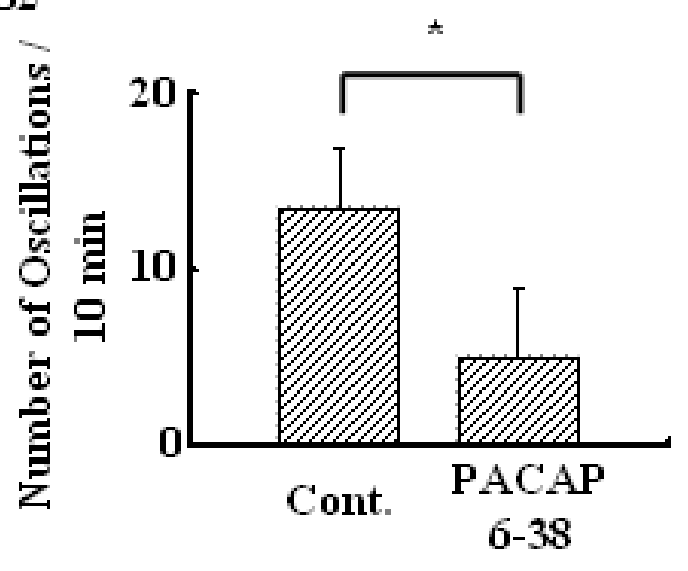

D

anti-GLT-1
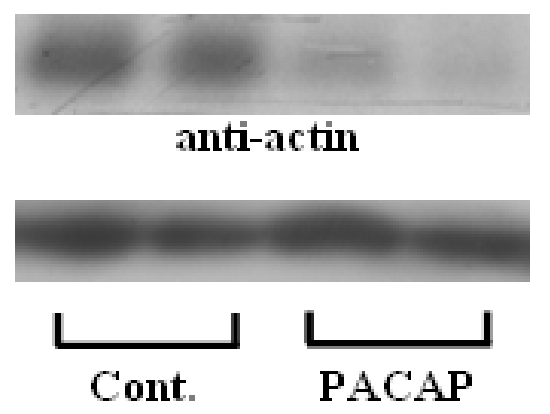

E

6-38

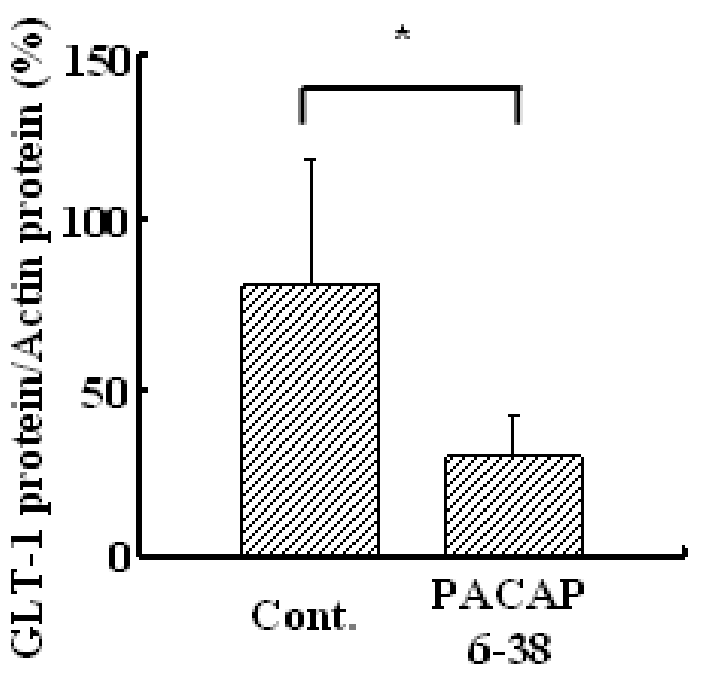


Neuron

Al

Ning

Astrocyte

A3

0.2

(F/FO)

1 min

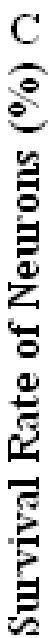

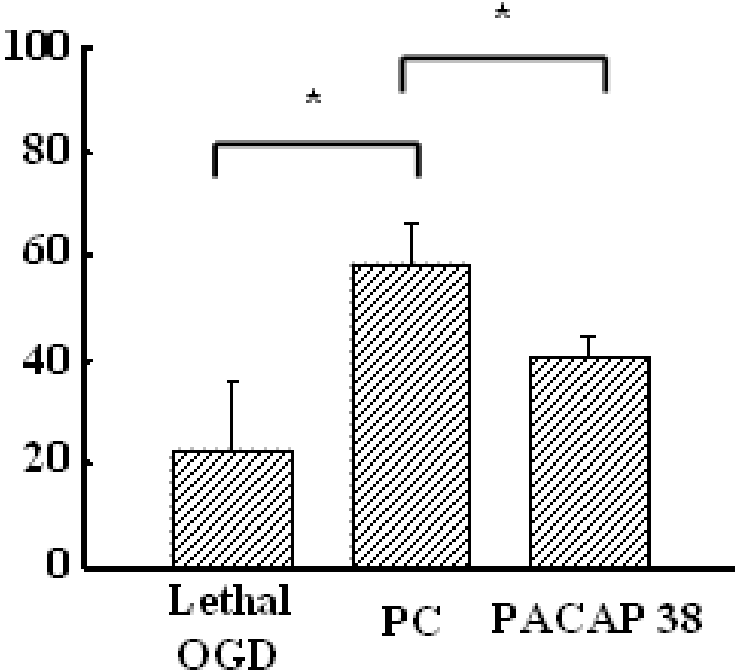

A2

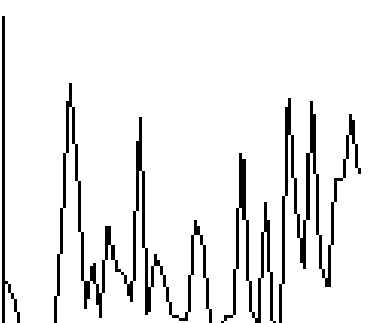

$\overline{100 \text { nM-PACAP } 38}$

A4

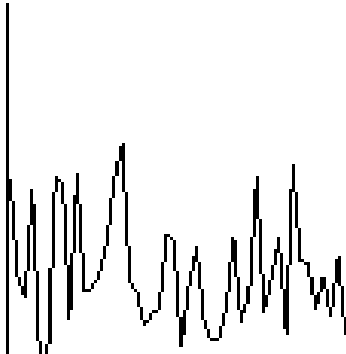

$\overline{100 \text { nM-PACAP } 38}$
D

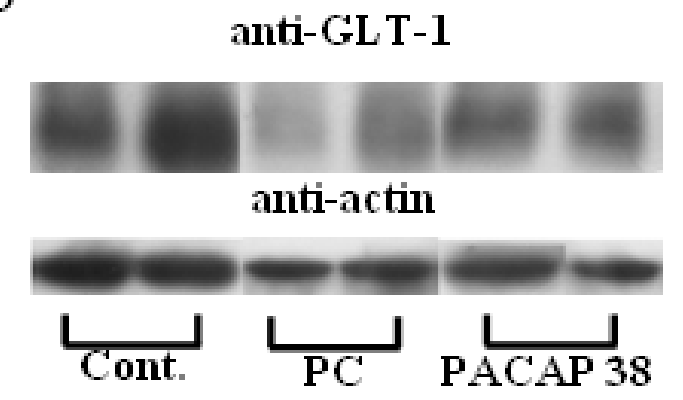

E

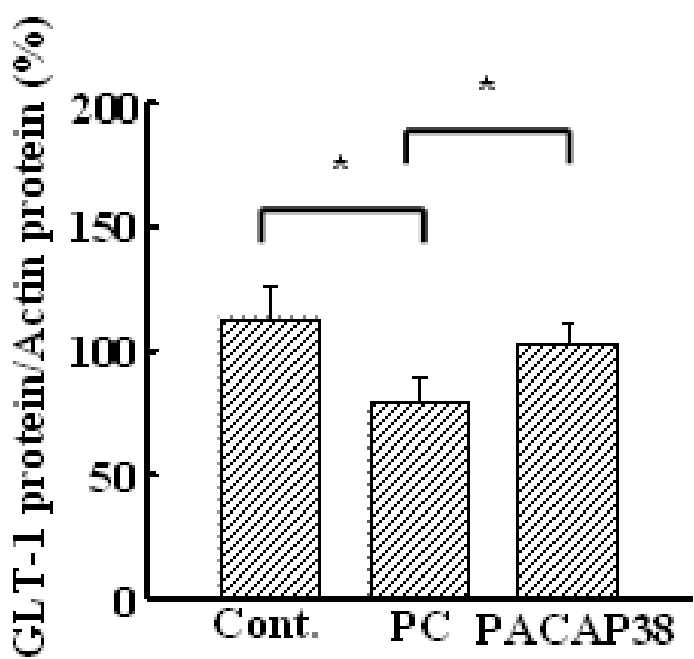


Neuron

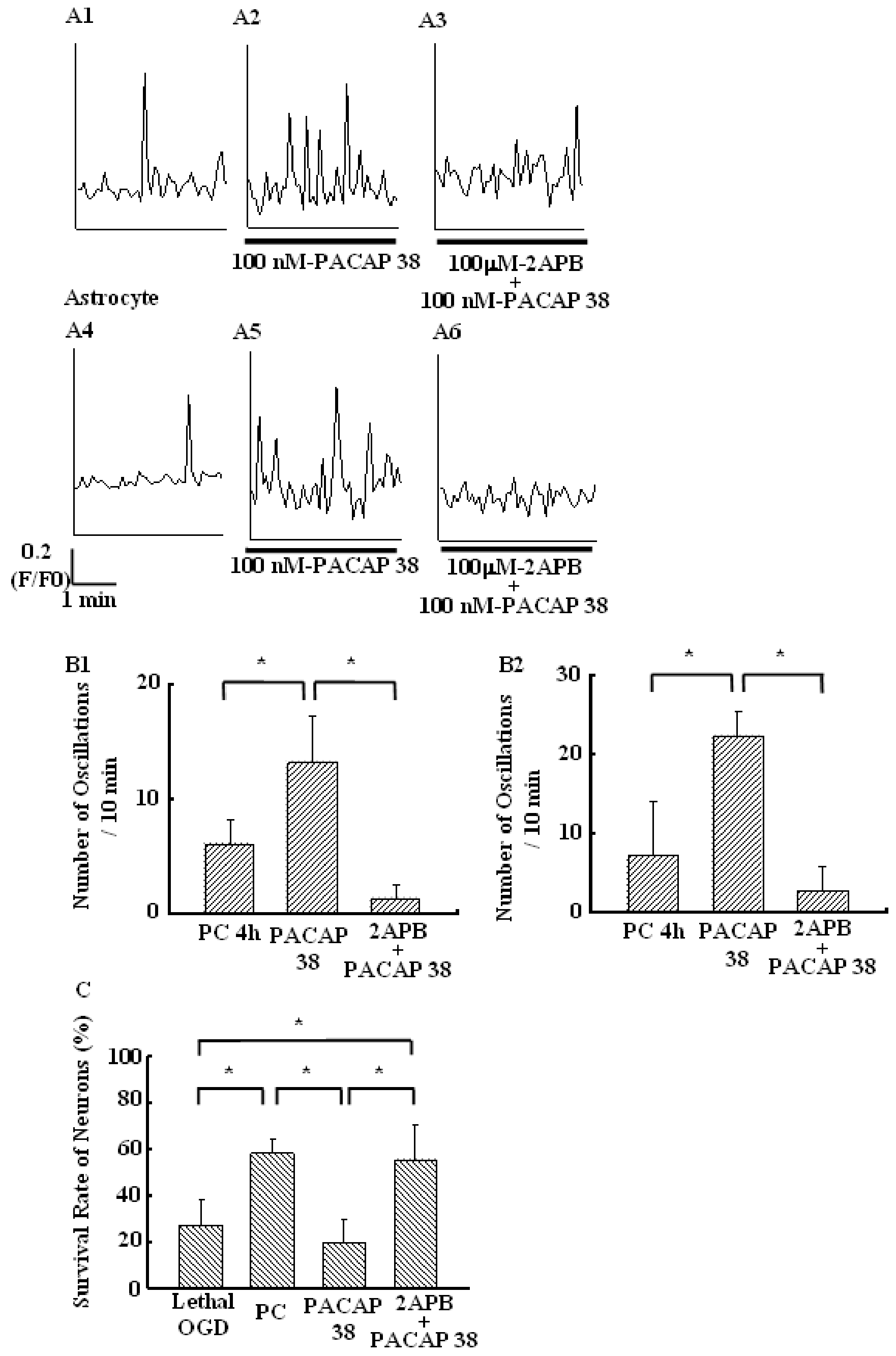

